Revue belge de géographie

1 | 2008

Aspects of the history of the Belgian geography and cartography

\title{
Géographie et éducation esthétique. Les estampes scolaires de la Ville de Bruxelles
}

Henri Nicolaï

\section{(2) OpenEdition}

Journals

Édition électronique

URL : http://journals.openedition.org/belgeo/10178

DOI : $10.4000 /$ belgeo.10178

ISSN : 2294-9135

Éditeur :

National Committee of Geography of Belgium, Société Royale Belge de Géographie

Édition imprimée

Date de publication : 31 mars 2008

Pagination : 47-68

ISSN : 1377-2368

\section{Référence électronique}

Henri Nicolaï, « Géographie et éducation esthétique. Les estampes scolaires de la Ville de Bruxelles », Belgeo [En ligne], 1 | 2008, mis en ligne le 19 octobre 2013, consulté le 01 mai 2019. URL : http:// journals.openedition.org/belgeo/10178; DOI : 10.4000/belgeo.10178

Ce document a été généré automatiquement le 1 mai 2019.

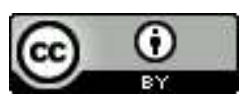

Belgeo est mis à disposition selon les termes de la licence Creative Commons Attribution 4.0 International. 


\title{
Géographie et éducation esthétique. Les estampes scolaires de la Ville de Bruxelles
}

\author{
Henri Nicolaï
}

1 Pendant la première moitié du $\mathrm{XX}^{\mathrm{e}}$ siècle, des écoliers belges, surtout dans l'agglomération bruxelloise, ont eu, sous les yeux, dans leurs classes, leurs couloirs et leurs préaux, de grandes chromolithographies représentant vingt-et-un paysages de leur pays. Cette imagerie géographique est intéressante à plus d'un titre. Elle montre d'une part la perception que l'on avait des paysages à cette époque. Elle semble témoigner d'autre part que l'on accordait une importance indéniable à la géographie dans la formation des élèves. Elle amène aussi à se poser une question d'ordre général. Faut-il y voir une utilisation de la géographie pour renforcer le sentiment national ?

Dans son étude sur l'histoire de la formation du sentiment national belge, notamment dans le volume 2 consacré au grand siècle de la nationalité belge (le XIXe), Jean Stengers (2002) ne cite pas l'argumentation géographique. Quelques lignes évoquent le recours à l'art par le canal des grands tableaux historiques. Mais rien sur la géographie. Celle-ci ne semble pas avoir été retenue comme un ciment potentiel de l'unité nationale. Les manuels de géographie cependant ne manquent pas de descriptions flatteuses des beautés naturelles du pays et de ses grandes réalisations urbaines et industrielles. Mais peut-être la tendance à insister sur leur diversité a-t-elle affaibli l'affirmation de l'unité nécessaire au sentiment national.

3 Au moment où la série d'estampes, qui nous occupe, a été réalisée, c'est-à-dire au début du XXe siècle, la Belgique se sent rassurée sur son espérance de vie. Elle a surmonté les crises de 1848 et de 1870. La tempête de 1914 ne s'annonce pas encore. Le sentiment national est exalté dans le système scolaire par des chants patriotiques, par une imagerie historique dans laquelle le mouvement communal du moyen âge tient une grande place, par la glorification de grands personnages historiques mais aussi par des récits héroïques sur des militaires belges au Congo et enfin par la ferveur patriotique qui a soutenu l'expédition antarctique de la Belgica. 
4 L'exécution de la série d'estampes consacrées à des paysages de Belgique s'inscrit-elle ainsi dans un mouvement d'exaltation du territoire national et de la patrie? Cette motivation ne peut être complètement exclue. Elle apparait d'ailleurs dans le discours publicitaire de la brochure des imprimeurs-éditeurs qui appelle à la souscription (figure 1). Cette série, écrivent-ils, "réalise une belle et grande glorification de la terre belge et de ce que l'activité de ses habitants a su en faire. Dans les écoles, auxquelles ces planches sont destinées, elles contribueront puissamment à faire connaître aux élèves les différents aspects et les beautés de la Patrie [ces derniers mots sont imprimés en grand et en caractères gras] en même temps qu'elles feront oeuvre de bonne éducation et de patriotisme".

Figure 1. Pages 1 et 4 de la brochure publicitaire de la firme O. De RYCKER et MENDEL.

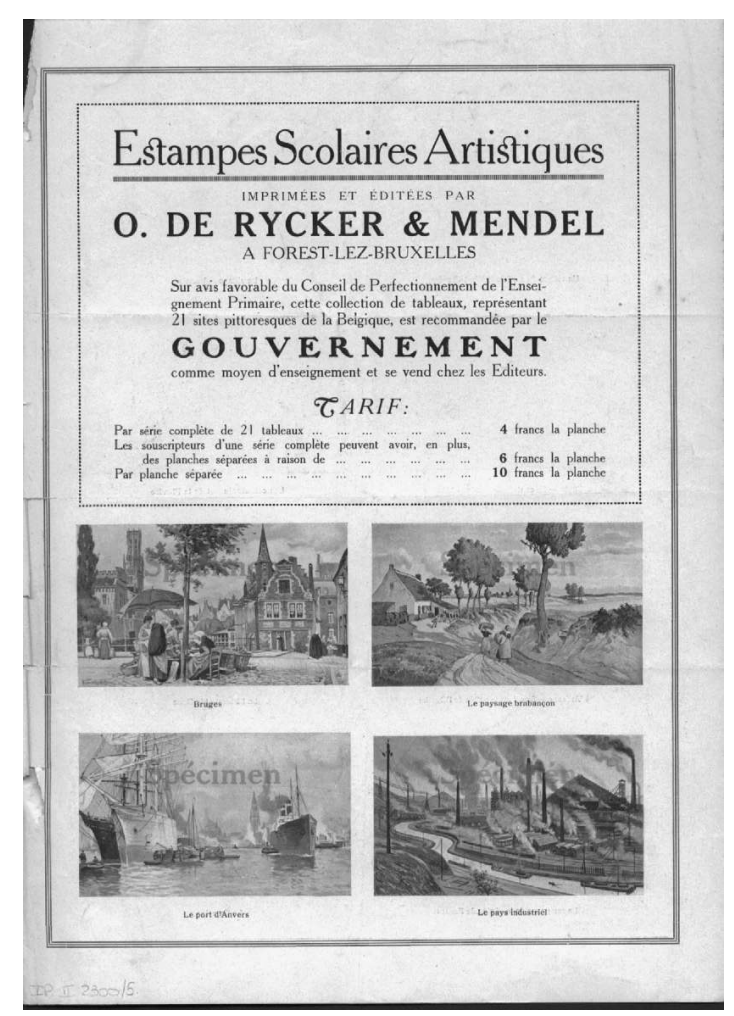

5 Cette brochure date de 1911 au plus tôt puisqu'elle comporte une estampe réalisée seulement cette année-là (celle de Pierre Paulus sur le paysage industriel). Archives de la Ville de Bruxelles, IP II 2300/5.

Des exemplaires des planches du village du nord de la West-Flandre (H. Cassiers) et de la vallée de la Meuse (F. Toussaint) avaient été envoyés comme échantillons dès 1908 aux souscripteurs potentiels.

7 Mais au départ, ce type de motivation n'est pas mis en avant par les concepteurs de la série et n'est jamais présenté comme l'objectif principal. Celui-ci est défini d'abord comme étant essentiellement d'ordre esthétique. D'ailleurs, même quand la série sera distribuée dans les écoles, une circulaire de l'échevin de l'Instruction publique de la Ville de Bruxelles, rappellera aux enseignants que c'est à l'initiation à l'art et au bon goût que ces estampes sont d'abord destinées. C'est seulement après que les écoliers les auront longtemps contemplées, qu'on pourra poser quelques questions simples "pour comprendre le sujet" et "les amener à sentir la beauté de la scène ou du site reproduit". 
Lors des excursions scolaires, on fera allusion aux estampes représentant les lieux parcourus (Archives de la Ville de Bruxelles, désignées dans la suite de notre texte par l'abréviation $\mathrm{AB}$, voir dossier IP I 2300,1). Cependant les deux objectifs (l'esthétique et le patriotique) sont présents dans l'esprit de plusieurs membres de la Commission créée par la ville pour mettre sur pied le projet. Dans la lettre où il accepte d'en faire partie, A. Stassart (alias Georges Rency) écrit, par exemple: “J'applaudis vivement à cette idée qui aura pour double effet d'éveiller dans l'âme des enfants un sain patriotisme et le sentiment de l'admiration. Elle est aussi un grand pas fait vers l'ornementation de l'Ecole qui fut jusqu'à présent si froide et si nue et qui doit devenir peu à peu un endroit charmant, plein de lumière, de belles images et d'enfants joyeux" (A.B., dossier IP, II, 2300, 5).

8 L'idée de réaliser une série d'estampes en couleurs destinées aux écoles naît dans une petite brochure de 1903 écrite par des membres du corps enseignant de la Ville de Bruxelles, intitulée "L'éducation esthétique à l'école primaire" (AB, dossier IP II 2300, 1). Les auteurs préconisent de développer le sens esthétique des élèves en mettant sous leurs yeux des estampes en couleurs de grande qualité. Il est nécessaire en effet de leur donner au plus tôt de bonnes habitudes esthétiques. L'époque est d'ailleurs en faveur de la création d'écoles modernes qui auront non seulement des locaux bien aérés avec une architecture hygiénique et pédagogique mais qui devront être aussi des monuments artistiques comme le sont les hôtels de ville. Il leur faut une décoration intérieure faite de peintures murales et d'estampes d'art. L'estampe en couleurs apparait comme un des éléments le mieux adaptés. Ni la gravure ni la photographie de l'époque ne peuvent apporter en effet une initiation esthétique aux couleurs.

qui cherchent à décorer leurs locaux en utilisant notamment des affiches. Mais elles les mettent en garde contre ce matériel car l'affiche est "faite pour la rue, avec sa lumière intense" et est "destinée à solliciter fortement mais passagèrement le regard" ( $\mathrm{B} B$, dossier IP II 2300, 1). Il arrive même parfois que des instituteurs bien intentionnés placent sous les yeux des enfants des affiches de mauvais goût ou à la vue déplaisante comme celles relatives à la campagne antialcoolique (A. Sluys, 1907, p. 58).

10 On évoque des exemples pris à l'étranger. En 1897, une association a été fondée en Suède pour la décoration des écoles par la reproduction d'oeuvres existantes et par des demandes expresses faites à des artistes nationaux pour des estampes originales de sites pittoresques, d'intérieurs traditionnels, de scènes historiques, de légendes, etc. (A. Sluys, 1907, p. 58).

11 Vers 1905, la Ville d'Anvers fait décorer une dizaine de locaux scolaires par de jeunes artistes sortant de son Académie des Beaux-Arts et offre une prime chaque année à un lauréat. En 1906, un Congrès d'art public se tient à Bruxelles. Il émet un voeu relatif à la décoration des locaux scolaires. La Ville de Bruxelles crée alors une commission qui est chargée de définir une vingtaine de sites de Belgique, de choisir des artistes pour les représenter et de faire imprimer des estampes en couleurs qui seront vendues aux différentes administrations communales pour leurs écoles, en un grand nombre d'exemplaires et à un prix peu élevé. Cette commission est composée de personnalités communales dont l'échevin de l'Instruction publique Léon Lepage (par la suite Emile Jacqmain) et le directeur général de l'enseignement Mabille, de professeurs à l'Académie des Beaux-Arts, à l'Athénée royal (A. Stassart, qui est aussi écrivain sous le nom de Georges Rency et secrétaire de l'Association des écrivains belges) et aux écoles normales 
(Alexis Sluys, Alfred Hegenscheidt), de directeurs d'école primaire, d'un homme de lettres (Léon Dommartin alias Jean d'Ardenne), d'un typographe-lithographe (AB, dossier IP II $2300,6)$. On y trouve en tête Charles Buls dont on connaît la passion esthétique pour les monuments de Bruxelles où, lorsqu'il était bourgmestre, il a dirigé les travaux de restauration de la Grand-Place. Il proposera de faire la promotion de la série dans le bulletin de la Ligue de l'Enseignement. Alexis Sluys, le directeur de l'école normale d'instituteurs, concepteur initial du projet, qui, dans la revue L'Art Public, l'a présenté en 1907, a dû jouer un rôle important. Peut-être aussi Alfred Hegenscheidt, professeur de géographie à l'école normale d'institutrices, qui deviendra par la suite professeur à l'Université Libre de Bruxelles où il créera une vingtaine d'année plus tard l'Institut de Géographie

Les documents conservés aux archives de la Ville ne permettent pas de savoir la part respective des différents membres dans l'exécution du projet. Selon Marius Renard, c'est vraisemblablement Mabille, le directeur général de l'enseignement, qui a assumé le rôle le plus effectif (M. Renard, 1921, p. 150). On n'a que très indirectement des informations sur la façon dont les sujets ont été sélectionnés et éventuellement modifiés et sur la procédure suivie pour le choix des artistes.

Voyons ce que l'on peut dire cependant sur chacun de ces points.

Il a été décidé que l'on exécuterait des estampes originales. Certains avaient pensé au départ faire reproduire des oeuvres existantes. Hegenscheidt avait proposé par exemple un tableau d'Alfred Verwée et un autre de Constantin Meunier. L'idée fut rejetée, d'abord parce qu'il était difficile de trouver, pour tous les sujets prévus par la commission, des tableaux déjà exécutés et qui répondraient à ses objectifs pédagogiques et ensuite parce que la transposition en estampes de tableaux existants risquait d'en altérer la valeur esthétique.

On a retenu assez vite l'imprimeur-éditeur O. De Rycker et Mendel, de Forest avec lequel un contrat a été signé. C'est un spécialiste des affiches d'art, des chromolithographies et des chromotypographies artistiques. Il a proposé d'exécuter des estampes, en fait des chromolithographies, de $1 \mathrm{~m}$ sur 0,6 m, marges non comprises (dans la brochure publicitaire, $1,1 \mathrm{~m}$ sur $0,7 \mathrm{~m}$ ) au prix de 3 francs (ce sera par la suite 4 francs) à condition d'en tirer au moins un millier de séries complètes ( $A B$, dossier IP II 2306 c). Le prix est comparé à celui de 15 francs qui est demandé à ce moment pour une affiche de Henri Cassiers (qui sera un des principaux auteurs de la série). La réalisation des estampes devait durer plusieurs années (quatre en principe), ce qui permettait aux souscripteurs d'étaler la dépense dans le temps. Les estampes sont datées ainsi de 1907 à 1911.

D'autres imprimeurs ont certainement été sur les rangs. On trouve trace, dans les dossiers, d'une proposition de la firme Bulens, rue Houzeau, qui propose d'exécuter vingt sujets dont elle établit elle-même la liste (AB, IP, II, 2500).

17 La commission a composé la liste des 21 sites à représenter. Il est possible que le choix n'ait pas été entièrement son fait. L'imprimeur-éditeur a dû certainement donner son avis comme il le fera d'ailleurs lorsqu'il s'agira de choisir les artistes.

18 Voici les titres des 21 sujets retenus (sur les estampes, ces titres sont bilingues) tels qu'ils figurent dans la brochure publicitaire éditée par l'imprimeur, avec les noms des artistes qui les ont exécutés :

- Bruxelles. La Bourse (Fernand Toussaint) (fig. 2)

- Huy (Victor Wagemakers) (fig. 10) 
- Le Port de Pêche d'Ostende (Henri Cassiers) (fig. 4)

- Gand et l'Horticulture (Florimond Van Acker) (fig. 6)

- Le Pays de Waes (Henri Cassiers) (fig. 15)

- Liége (Fernand Toussaint) (fig. 8)

- La Vallée de la Meuse (Fernand Toussaint) (fig. 20)

- La Fagne (Henri Meunier) (fig. 19)

- Furnes (Florimond Van Acker) (fig. 7)

- Un village du nord de la WestFlandre (Henri Cassiers) (fig. 14)

- Le village ardennais (Henri Cassiers) (fig. 22)

- Thuin. La contrée de Sambre et Meuse (MarcHenri Meunier) (fig. 11)

- Les Collines du sud de la Flandre (Henri Cassiers) (fig. 16)

- L’Ardenne. Site dans la vallée de l'Ourthe (Florimond Van Acker) (fig. 21)

- Le Littoral et la Dune (Henri Cassiers) (fig. 12)

- La campagne de la région de Furnes (Florimond Van Acker) (fig. 13)

- La Campine (Fernand Toussaint) (fig. 17)

- Bruges (Florimond Van Acker) (fig. 5)

- Le paysage brabançon (Amédée Lynen) (fig. 18)

- Le port d'Anvers (Henri Cassiers) (fig. 3)

- Le pays industriel (Pierre Paulus) (fig. 9). exécuter la série. Henri Cassiers se taille la part du lion avec sept estampes soit le tiers de l'ensemble. Il faut dire qu'il était un des meilleurs collaborateurs de la firme O. De Rycker. Ajoutons aussi, mais nous ne savons pas si cela est entré en ligne de compte, que son beau-frère, Léon Dommartin, était membre de la commission. Sous le pseudonyme de Jean d'Ardenne, ce dernier a écrit plusieurs guides touristiques dont un au moins a été illustré par Cassiers. Florimond Van Acker a été lui aussi très actif : cinq estampes. Fernand Toussaint en a exécuté quatre dont tout particulièrement celle de la Bourse à Bruxelles. une réputation internationale. Il est, par exemple, l'auteur d'une affiche de l'Exposition universelle de Bruxelles de 1910. Un éditeur new-yorkais a même acquis, au début du XX siècle, les droits sur un grand nombre de ses oeuvres dont notamment ses affiches des grandes compagnies transatlantiques (Koekelbergh I. et Pottier W., 1994, p. 11). Il a été un des illustrateurs d'Emile Verhaeren ; il a dessiné les paysages d'un livre sur le Brabant ; il est l'auteur d'une série de 12 cartes postales illustrées de stations balnéaires belges et de nombreuses cartes postales sur des villages des Pays-Bas avec leurs habitants parés de costumes régionaux. James Ensor l'a surnommé prince des aquarellistes pour sa participation à un salon d'Ostende consacré à des peintures de marine (Koekelbergh I. et Pottier W., 1944, p. 10).

Florimond Van Acker (Bruges 1858-1940) est un artiste brugeois qui a séjourné longtemps à Bruxelles avant de retourner en 1910 se fixer dans sa ville natale où il sera professeur puis directeur à l'Académie des Beaux-Arts. Spécialisé dans les paysages urbains, il a été un des animateurs de ce qu'on a appelé l'Ecole de Bruges.

Fernand Toussaint (Bruxelles 1873-1956), issu de l'Académie des Beaux-Arts de Bruxelles, est renommé pour ses portraits de grandes bourgeoises et ses nus féminins mais il est 
aussi l'auteur d'affiches des foires commerciales de Bruxelles et de l'Exposition universelle de Liège de 1905.

Henri (dit Marc Henry) Meunier (Ixelles, 1873- Bruxelles, 1922), élève puis professeur à l'Académie d'Ixelles, est un aquafortiste et aussi un affichiste.

Victor Wagemakers (Ganshoren 1876-1953) est connu pour ses paysages des campagnes brabançonnes et anversoises.

Amédée, Ernest Lynen (St-Josse-ten-Noode, 1852- Bruxelles, 1938), aquarelliste, lithographe décorateur, a illustré les oeuvre de Charles De Coster et a décrit souvent des scènes populaires. Il a réalisé pour l'Exposition universelle de Liège, en 1905, une grande fresque qu'Alexis Sluys aurait voulu voir acquérir par la Ville de Bruxelles pour orner une de ses écoles.

Pierre Paulus (Châtelet, 1881-Bruxelles, 1959) a réalisé de vigoureuses peintures sur le Pays Noir et ses travailleurs. Rappelons aussi, de façon anecdotique, qu'il est l'auteur du Coq qui figure aujourd'hui sur le drapeau de la Région wallonne.

Figure 2. Fernand TOUSSAINT. Bruxelles. La Bourse.

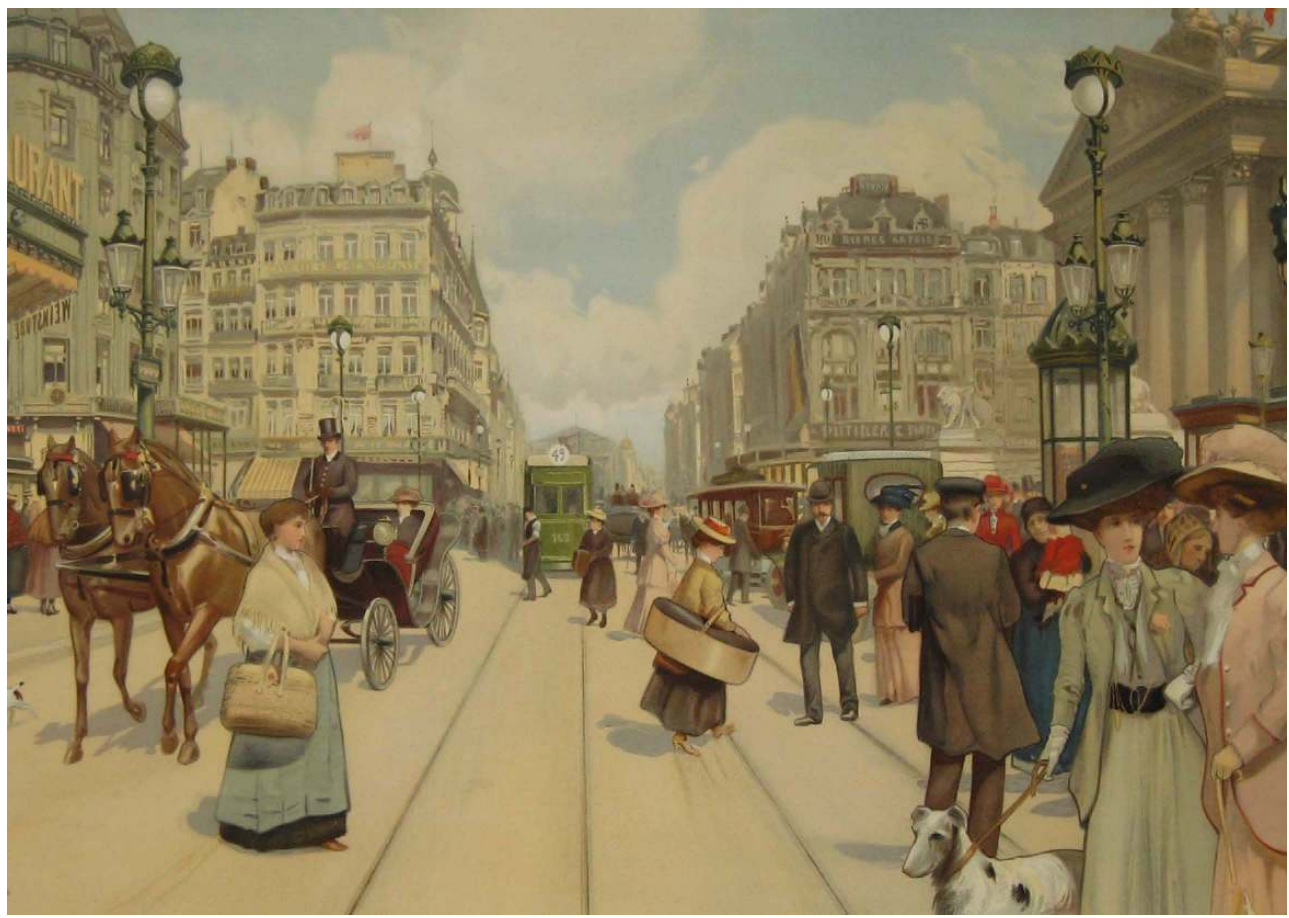


Figure 3. Henri CASSIERS. Le Port d'Anvers.

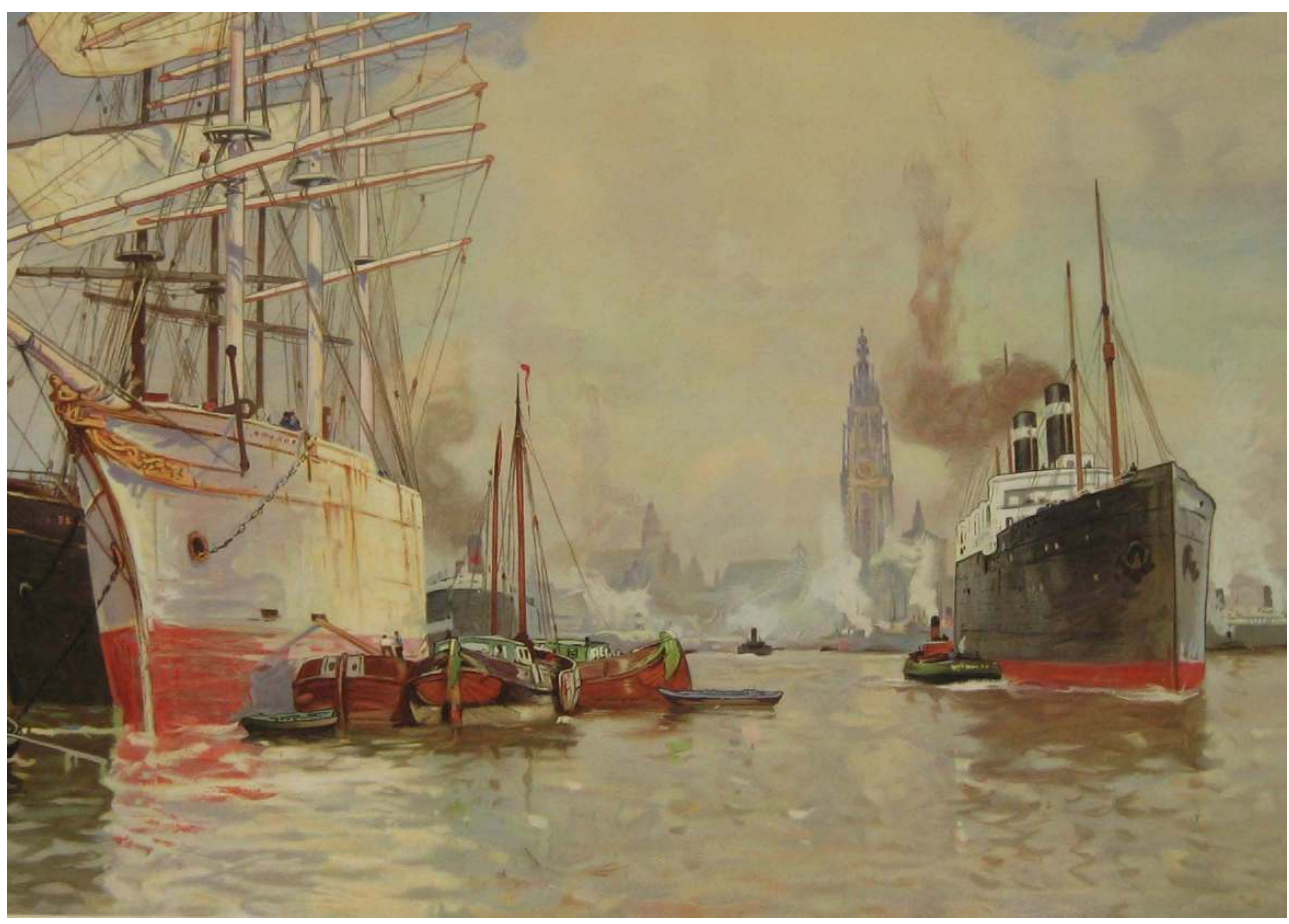




\section{Figure 4. Henri CASSIERS. Le Port de pêche d'Ostende.}

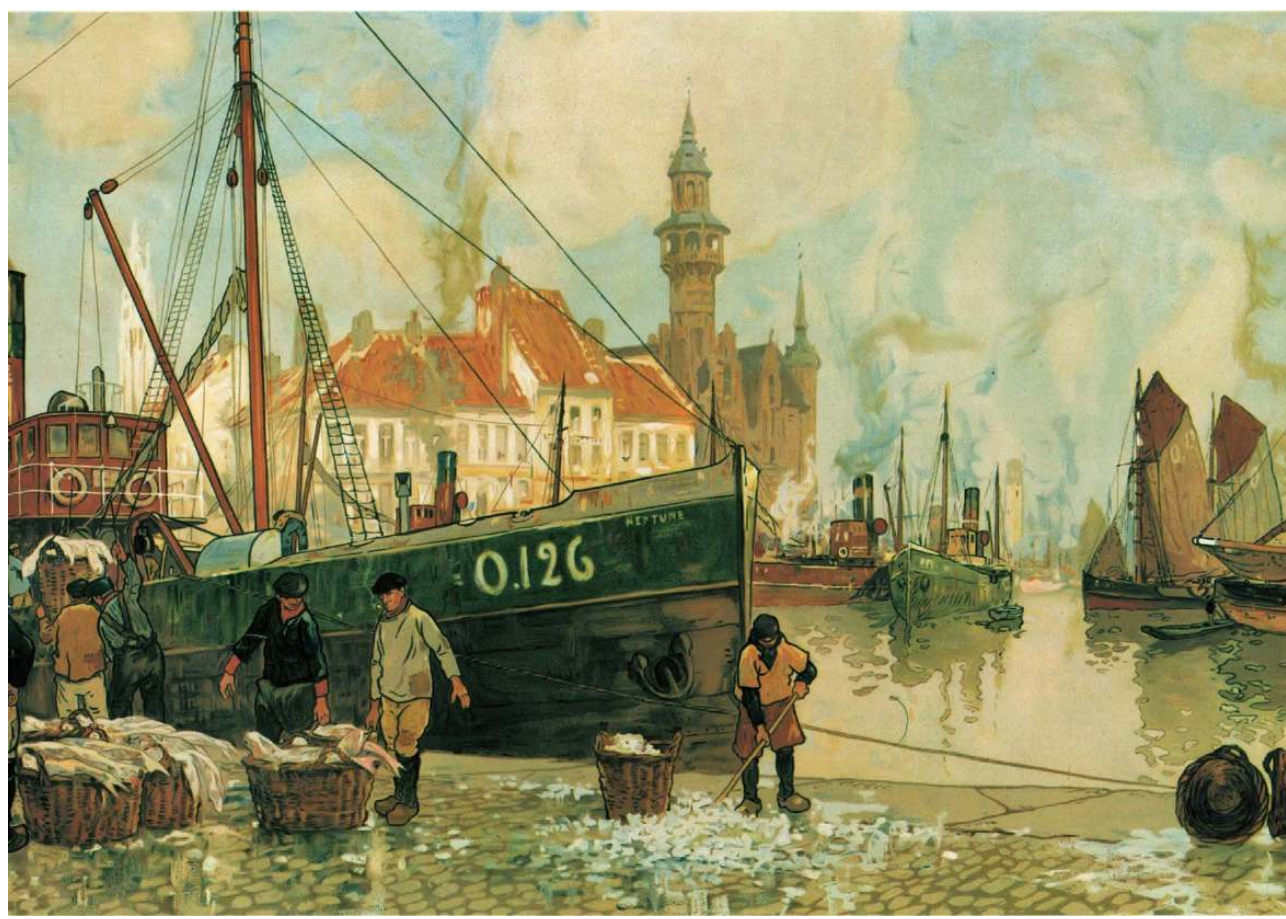

Les reproductions photographiques sont de Quentin Rombaux (figures 2, 3, 5, 6, 8, 9, 10, 11, 12, 13, 14 , $16,19,20,21$ ) et de Benjamin Wayens (figures 4, 7, 15, 17, 18, 22). Nous remercions Mme Gwendolyne Denhaene, du Cabinet des Estampes de la Bibliothèque Royale de Belgique, de nous avoir permis de les effectuer. Nous remercions aussi, pour son aide, M. Jean-Philippe Colombie, de la même institution. Nous remercions vivement Mme Marie-Laurence Bernard, de la boutique "Ma Maison de Papier" pour nous avoir permis de consulter des estampes que, par suite d'une lacune dans le catalogue, nous n'avions pu trouver au Cabinet des Estampes.

Les photos des estampes peuvent être consultées sur le site de la Société royale belge de Géographie www.srbg.be 
Figure 5. Florimond VAN ACKER. Bruges.

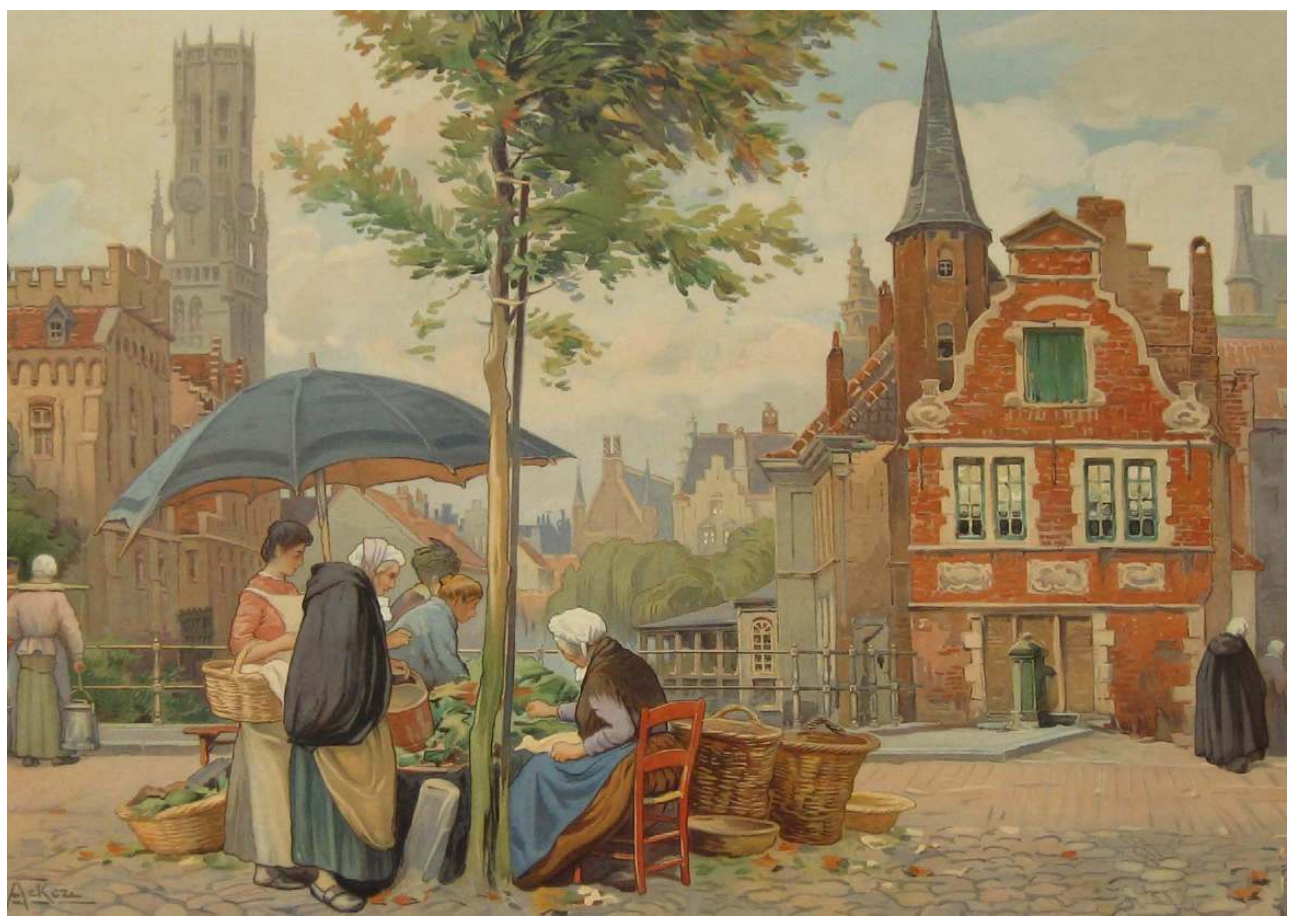

Figure 6. Florimond VAN ACKER. Gand et l'horticulture.

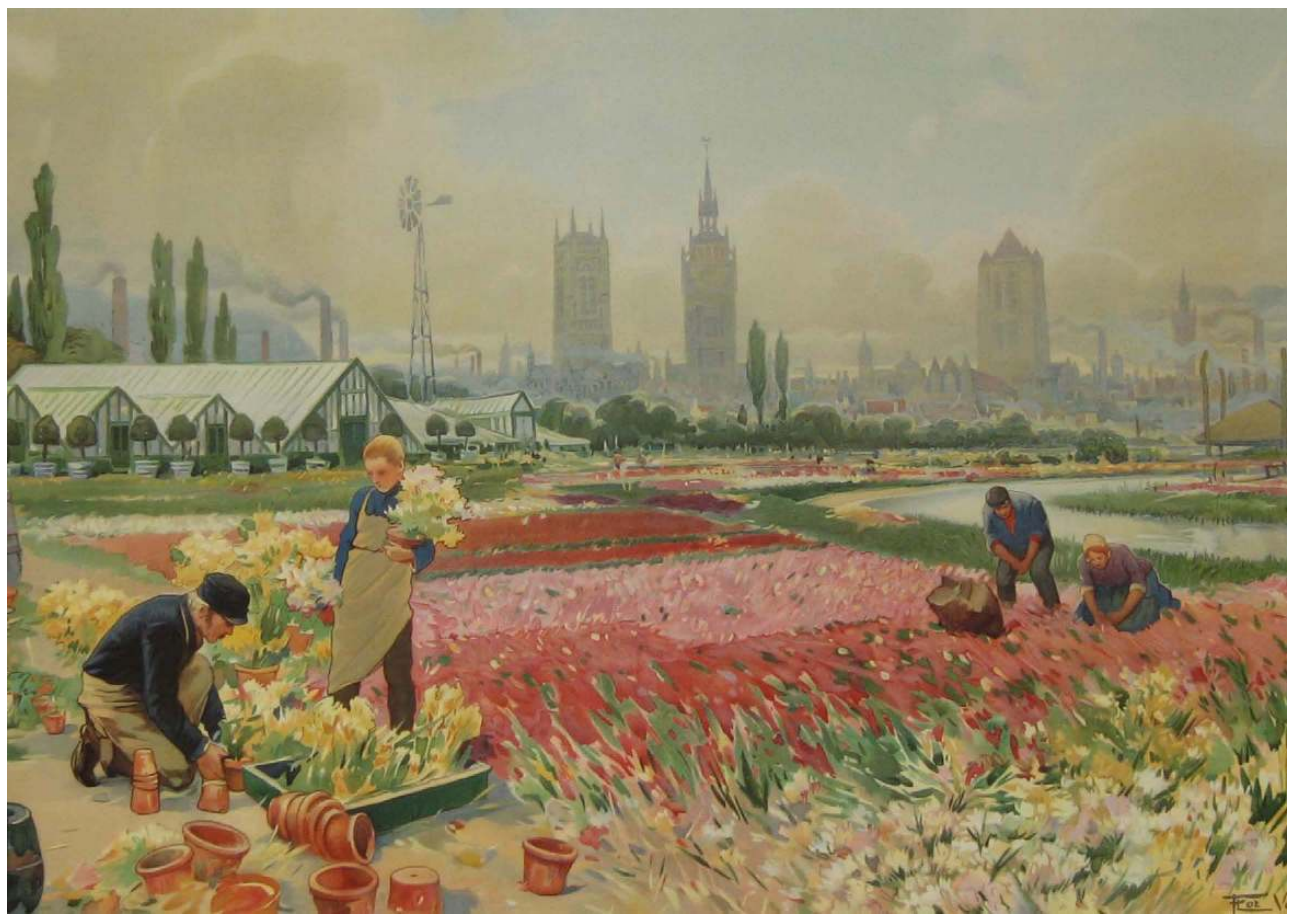


Figure 7. Florimond VAN ACKER. Furnes.

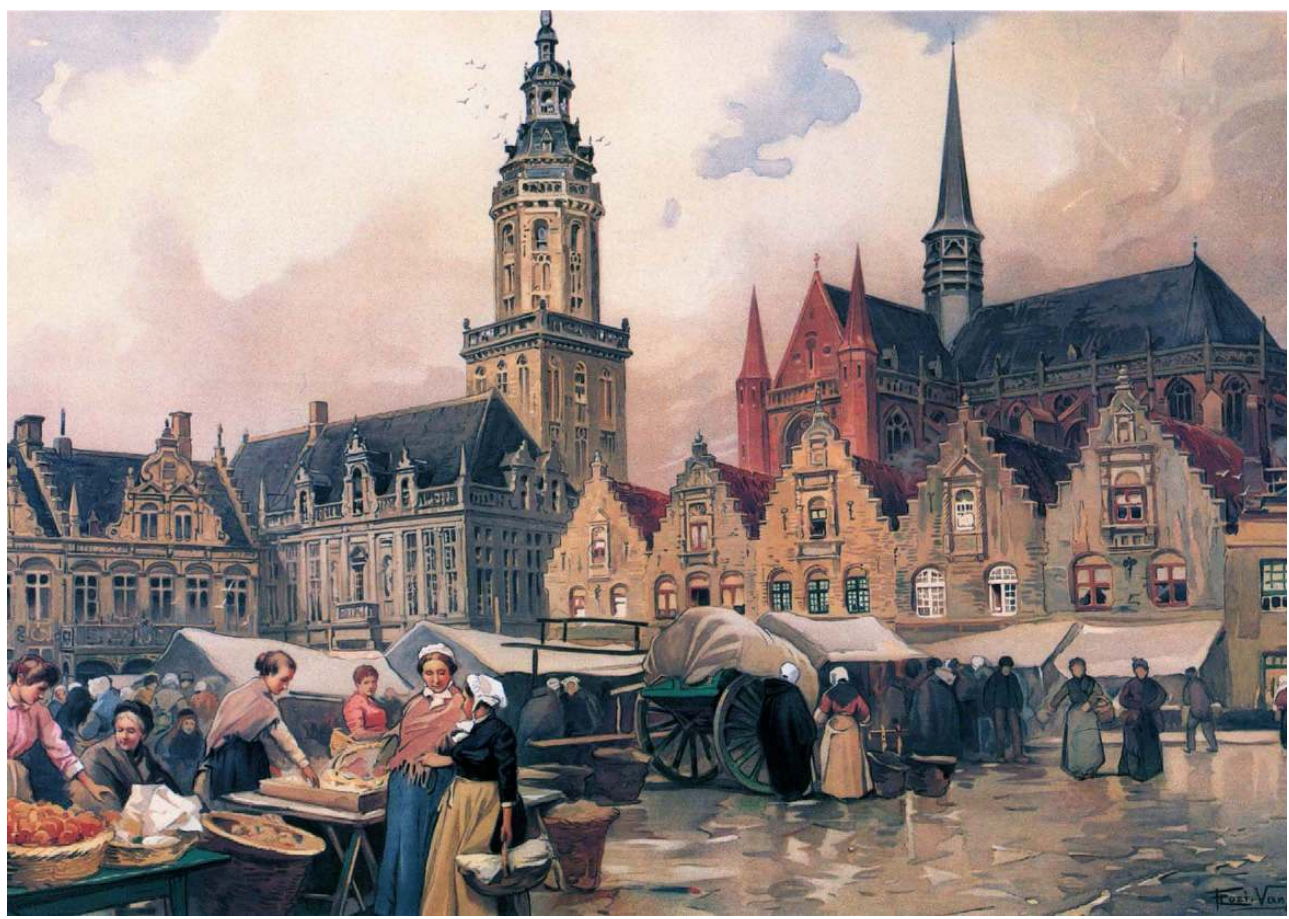

Figure 8. Fernand TOUSSAINT. Liége.

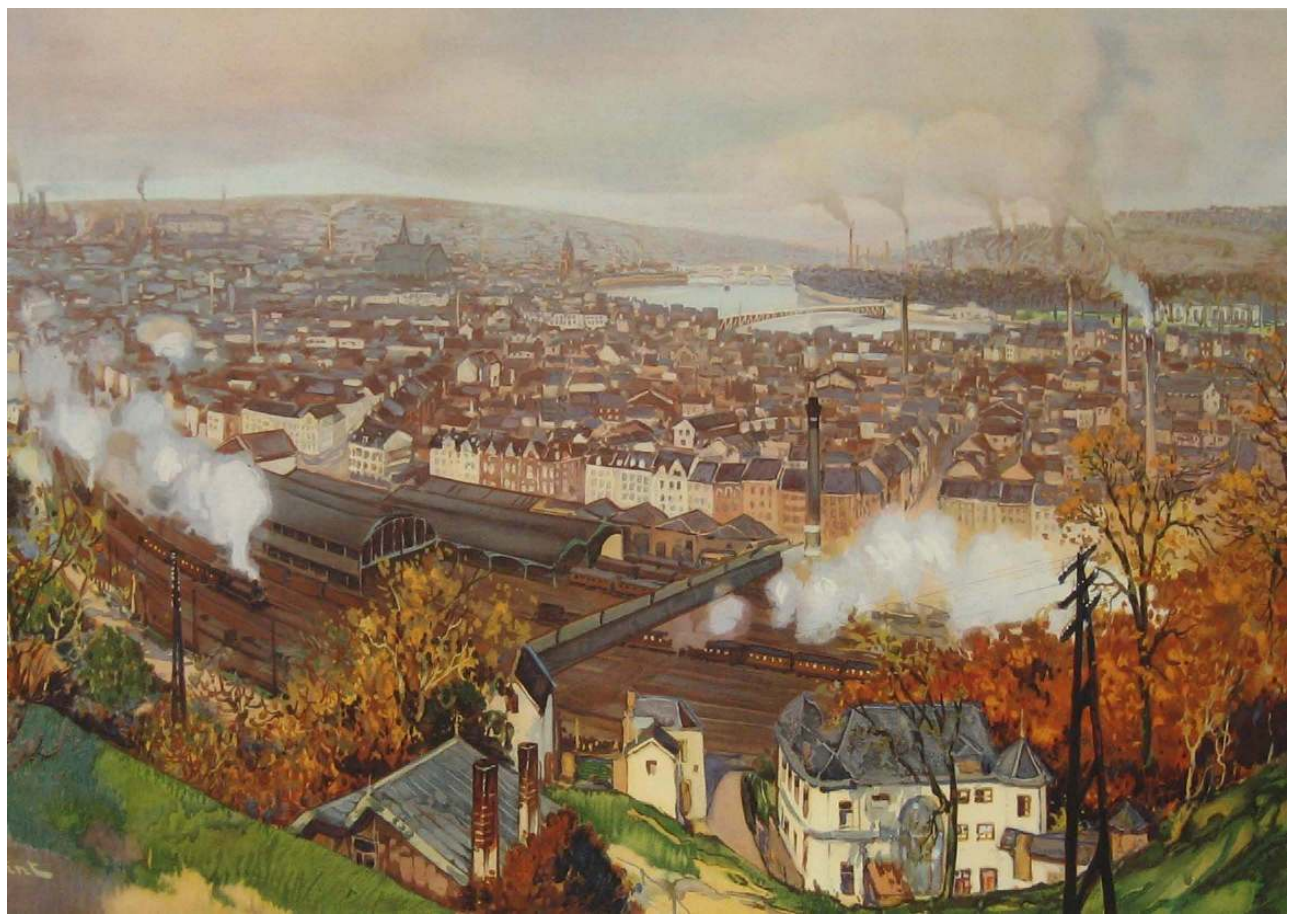


Figure 9. Pierre PAULUS. Le pays industriel.

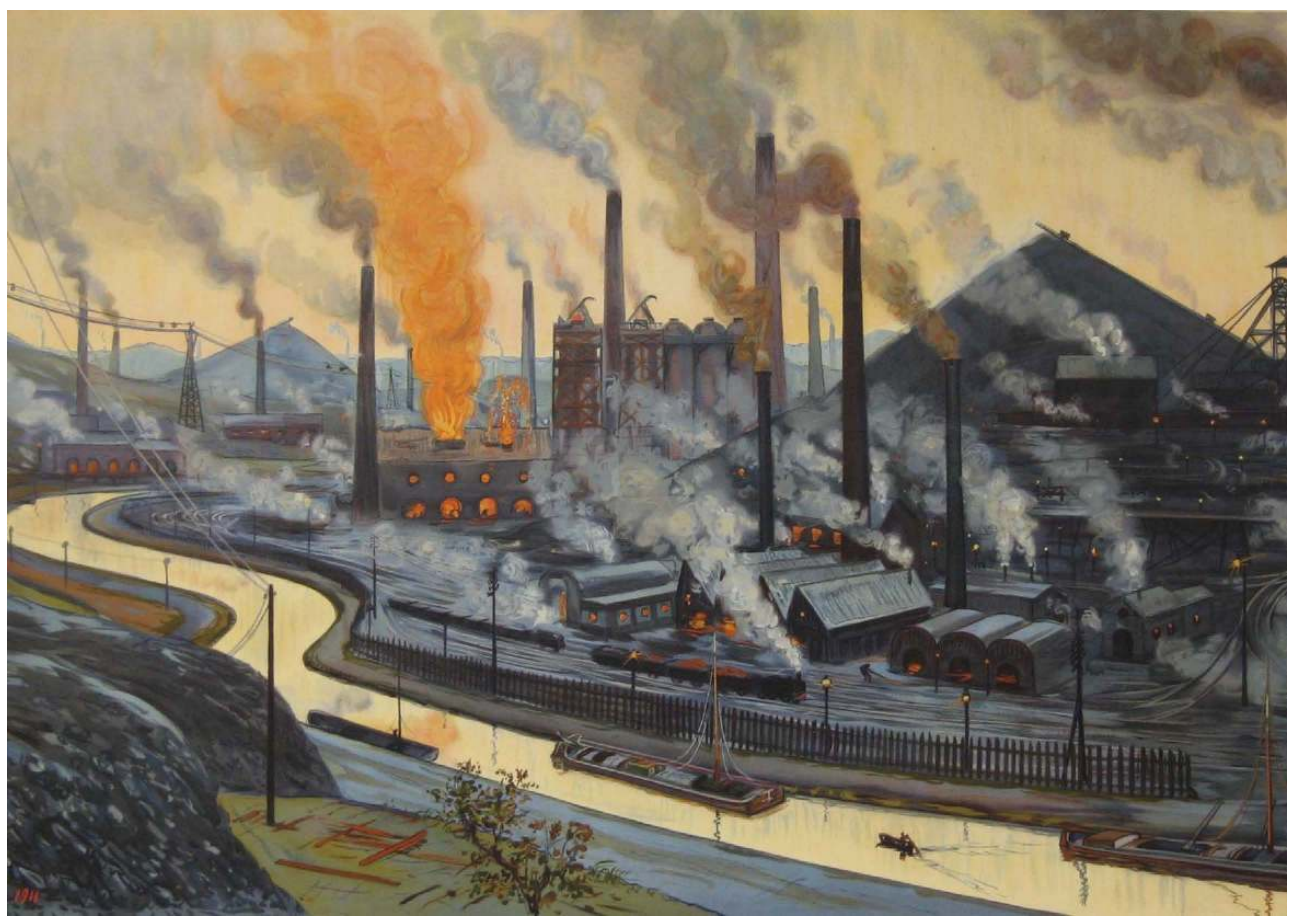

Figure 10. Victor WAGEMAKERS. Huy.

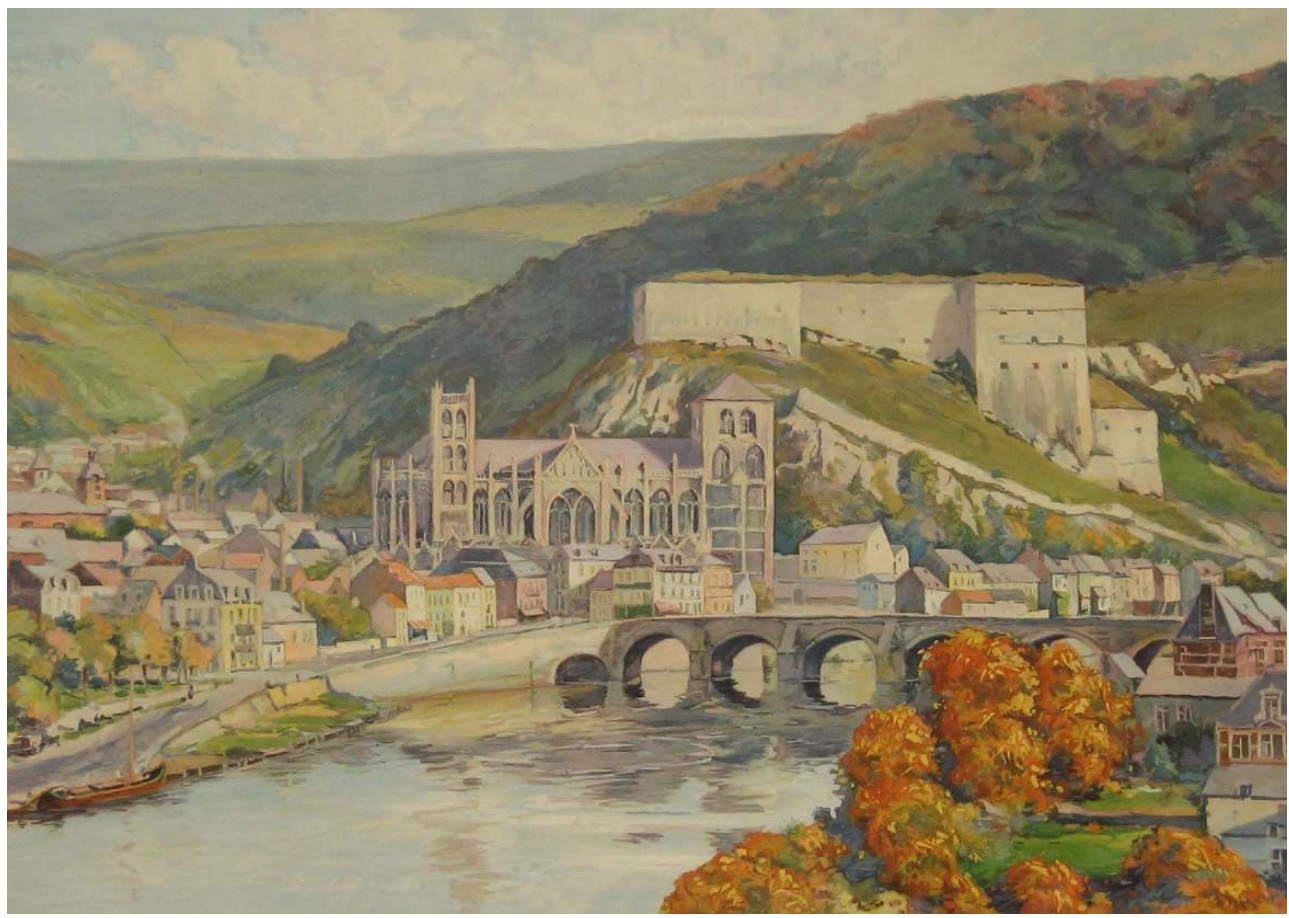


Figure 11. Henri MEUNIER. Thuin. La contrée de Sambre et Meuse.

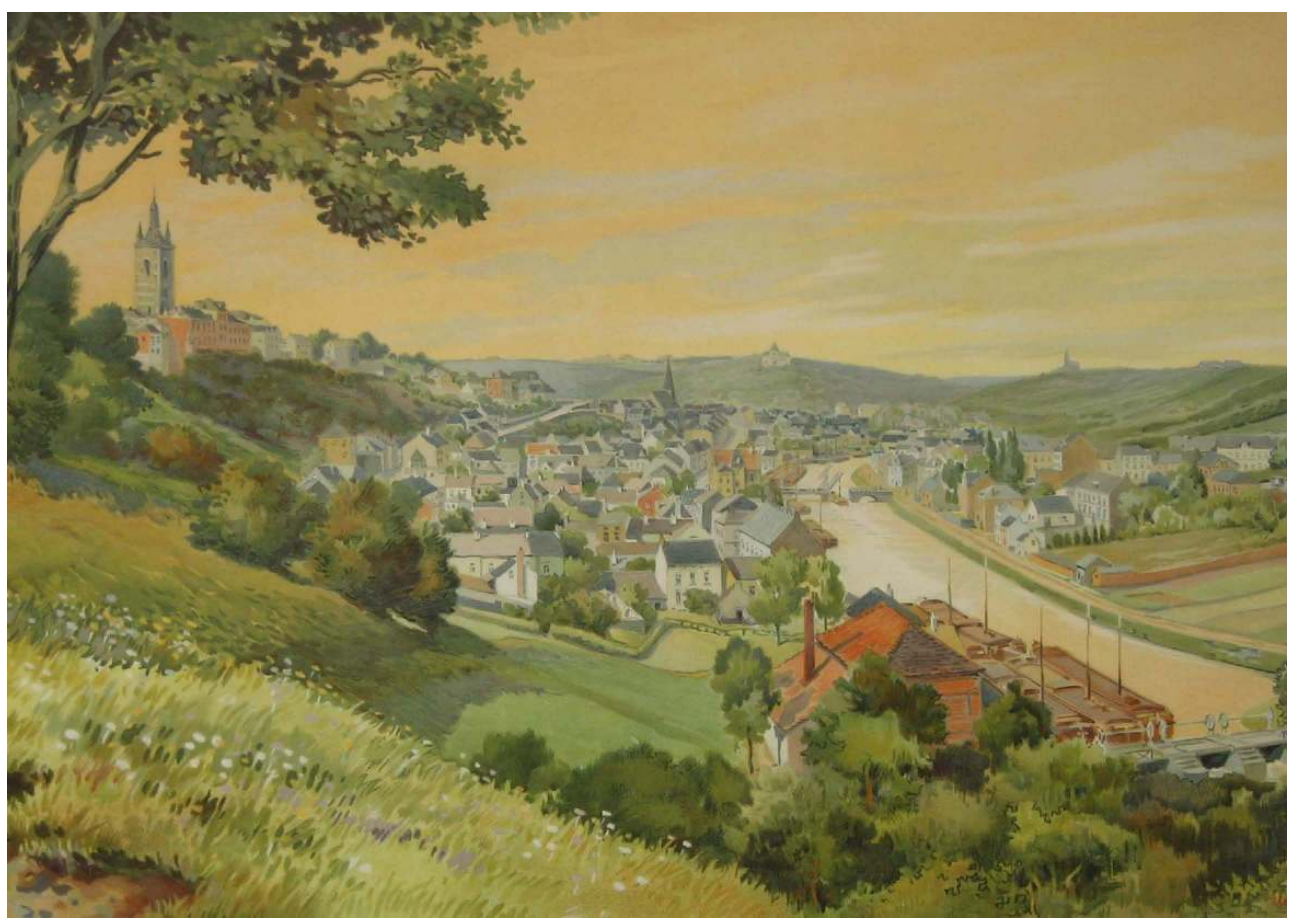

Figure 12. Henri CASSIERS. La Littoral et la Dune.

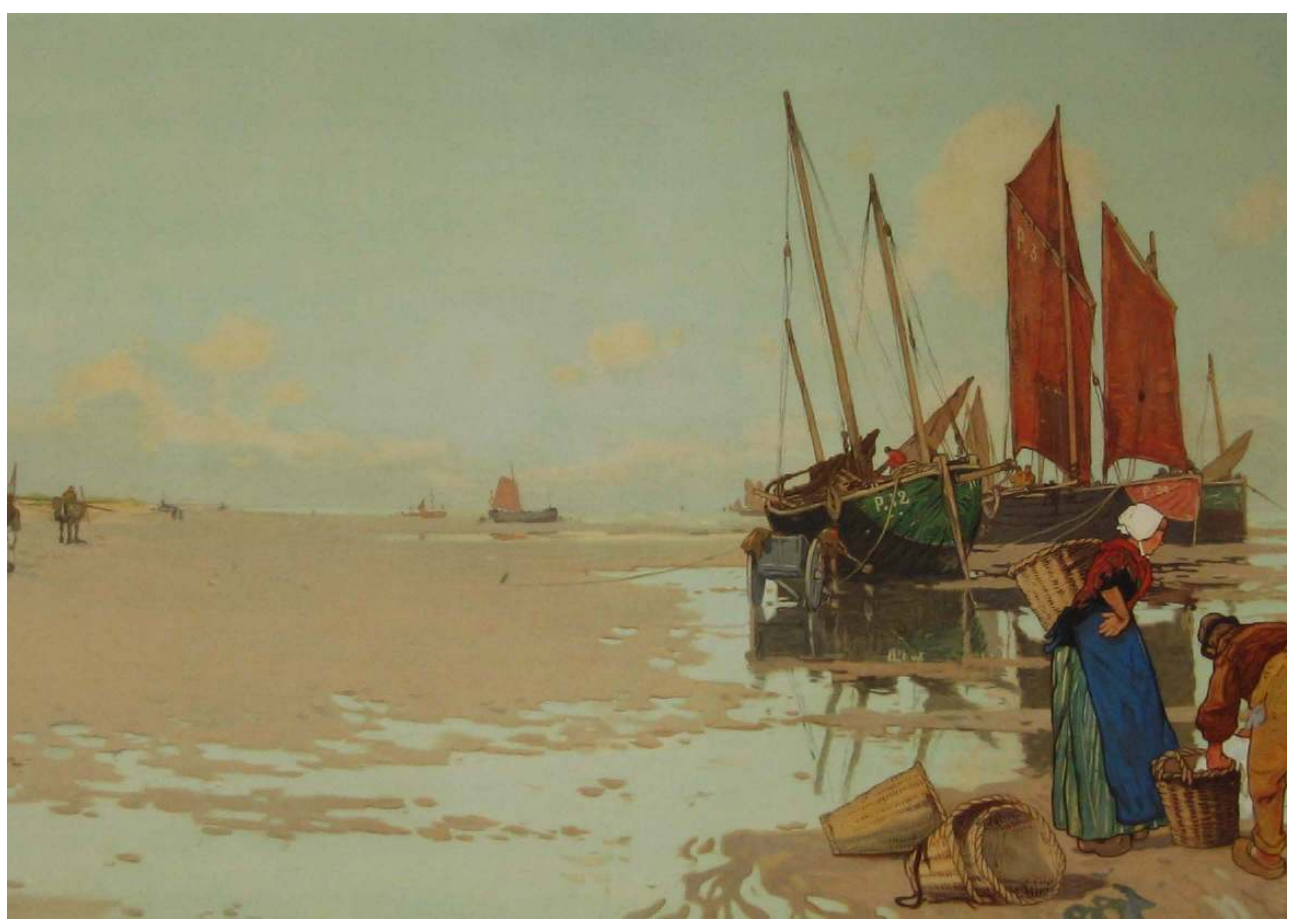


Figure 13. Florimond VAN ACKER. La camplagne de la région de Furnes.

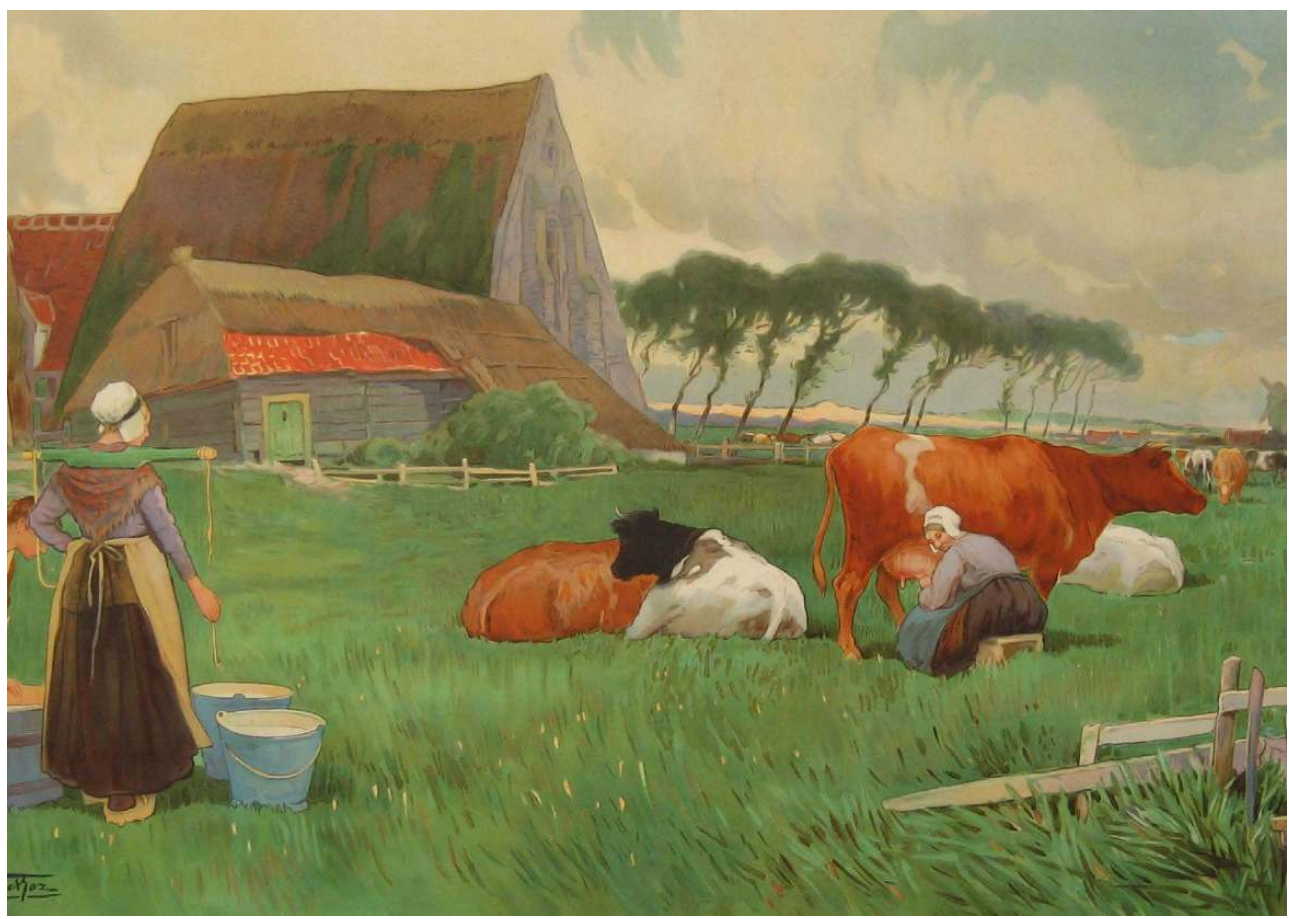

Figure 14. Henri CASSIERS. Un village du nord de la West-Flandre.

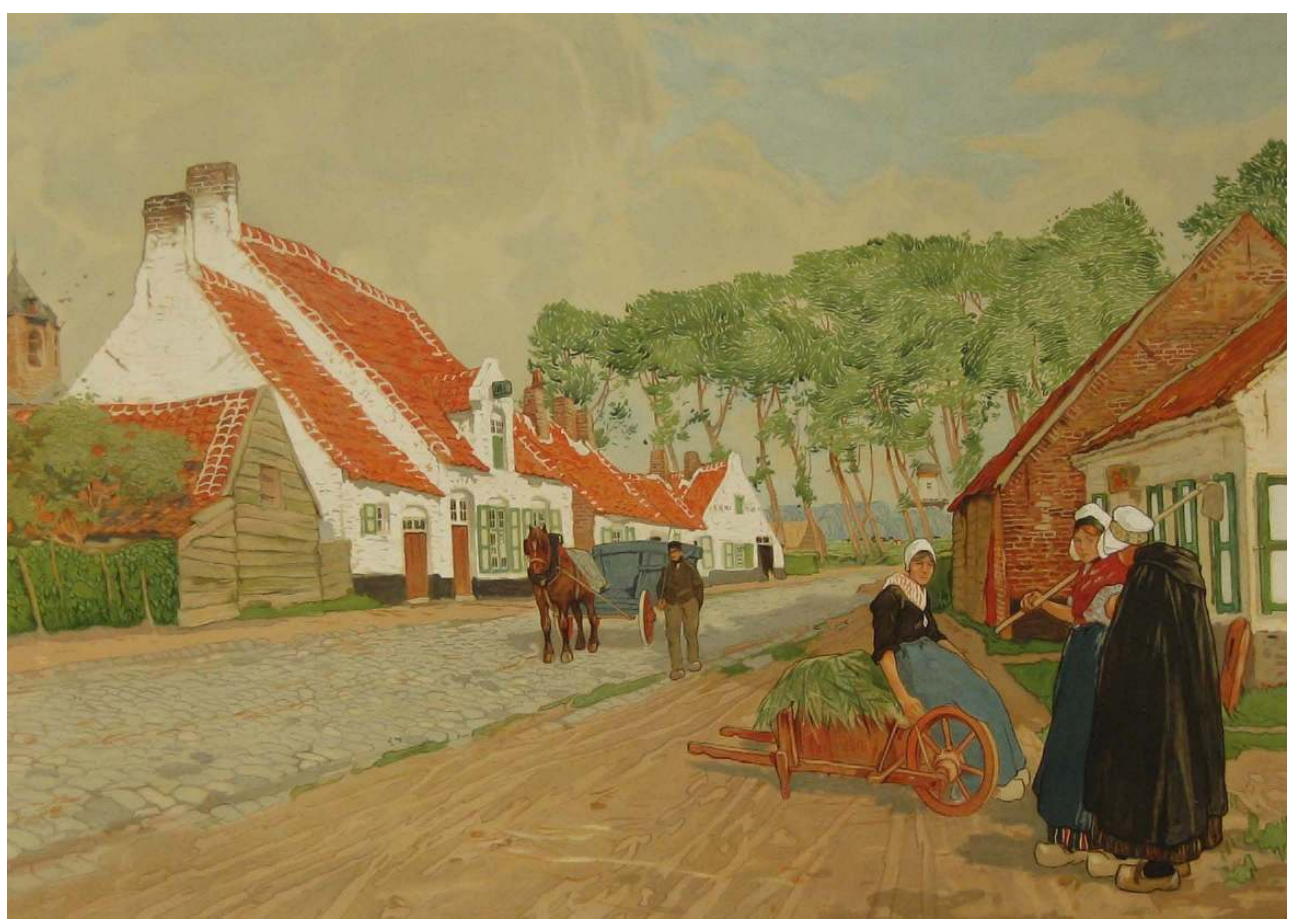


Figure 15. Henri CASSIERS. Le Pays de Waes.

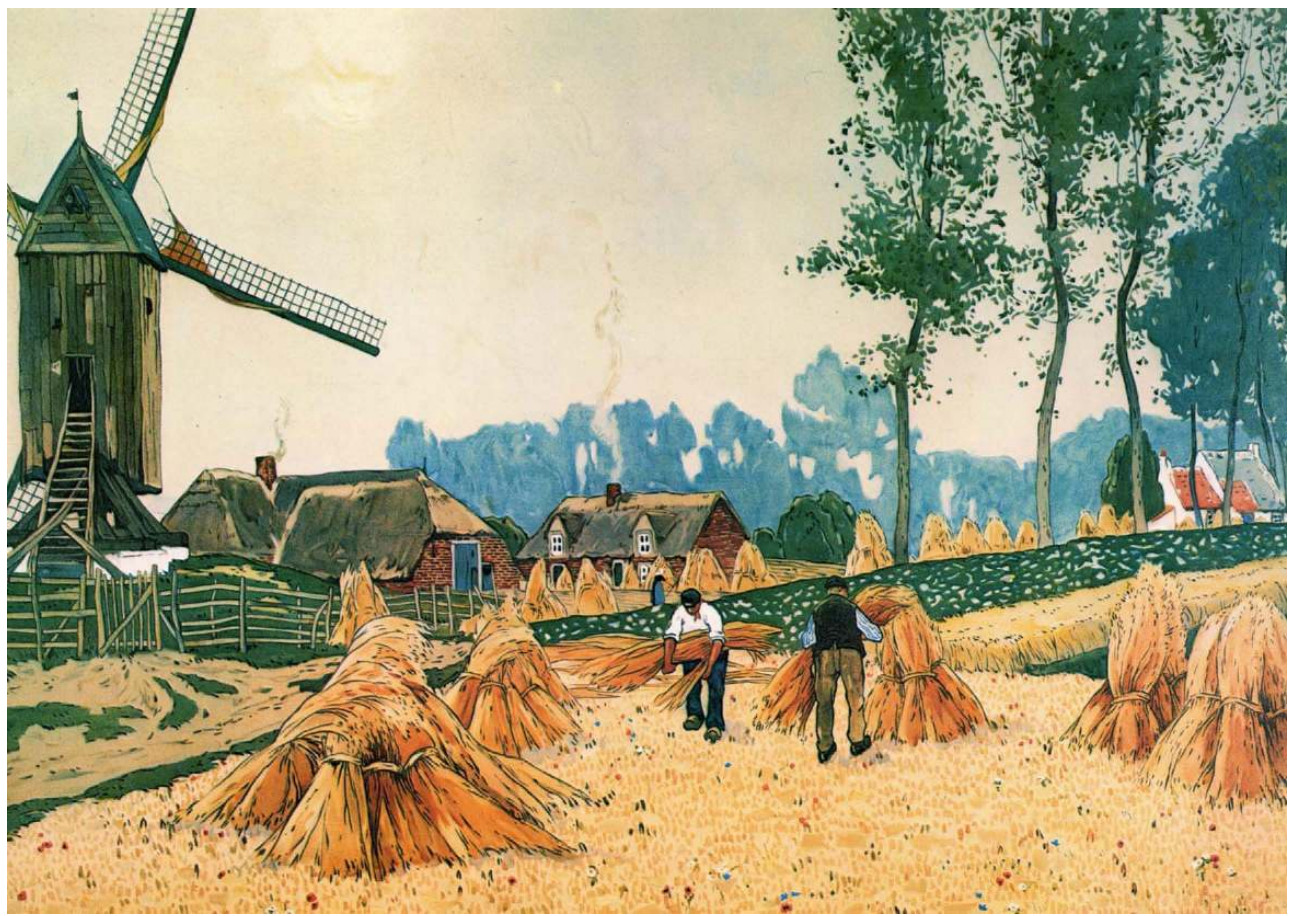

Figure 16. Henri CASSIERS. Les collines du sud de la Flandre.

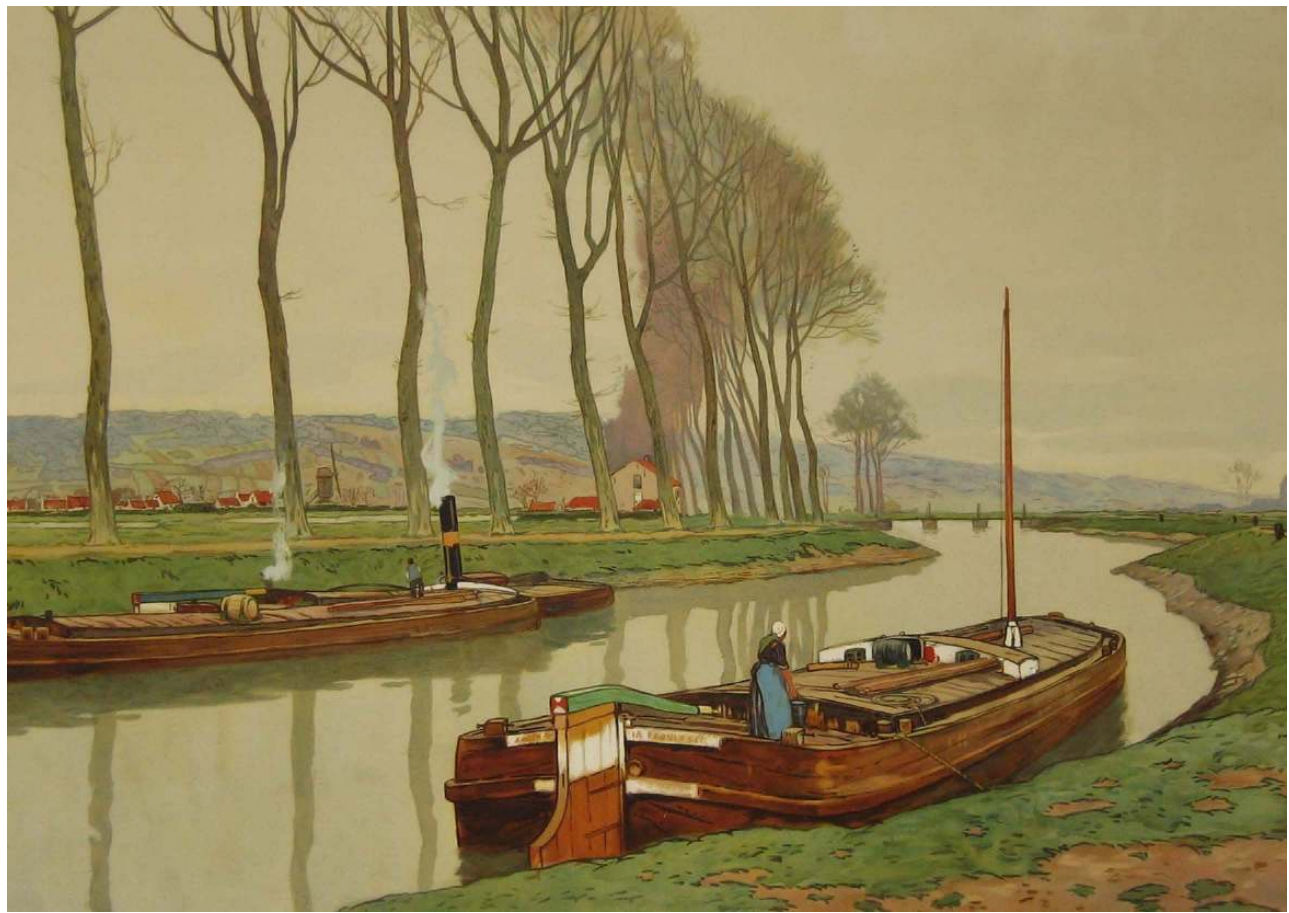


Figure 17. Fernand TOUSSAINT. La Campine.

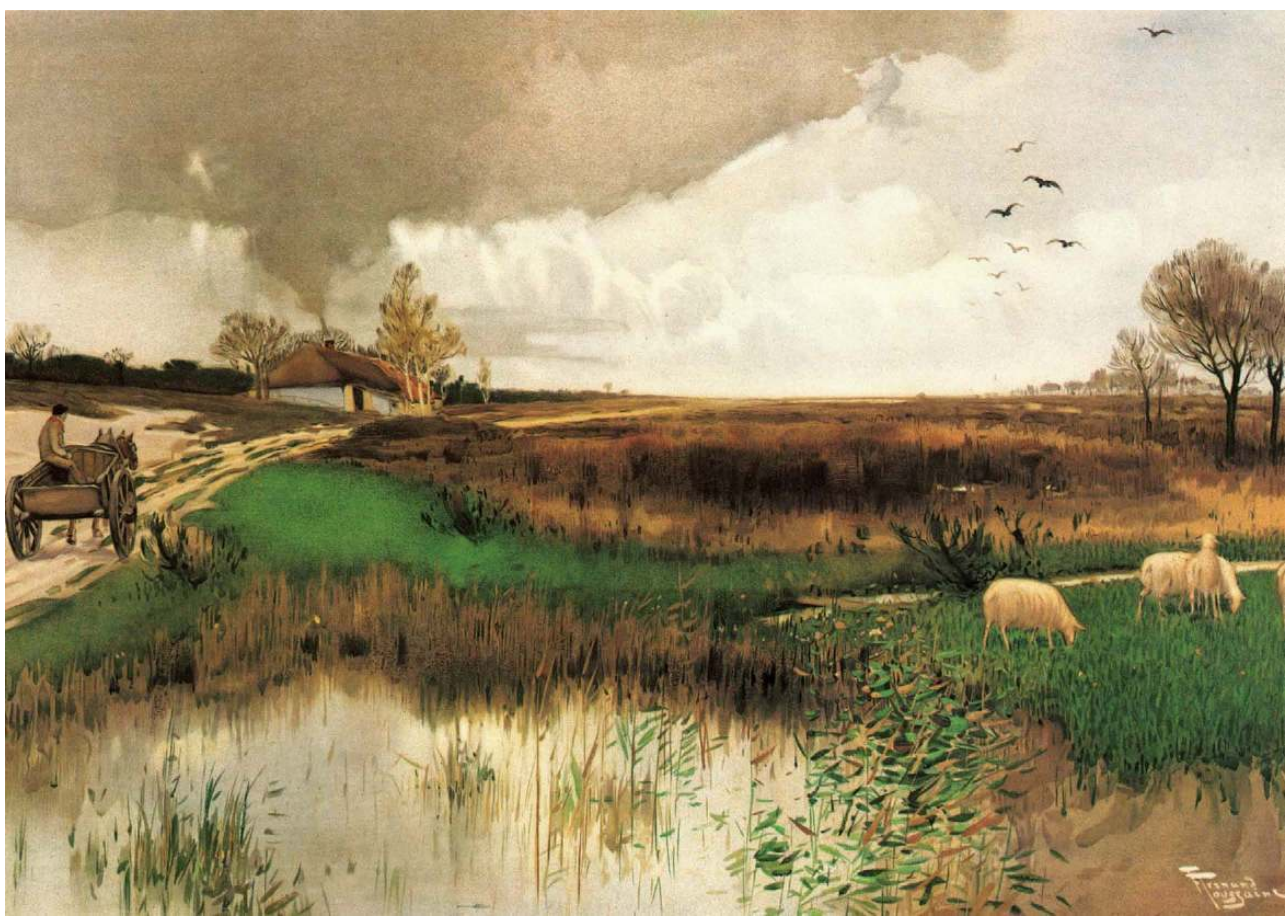

Figure 18. Amédée LYNEN. Le paysage brabançon.

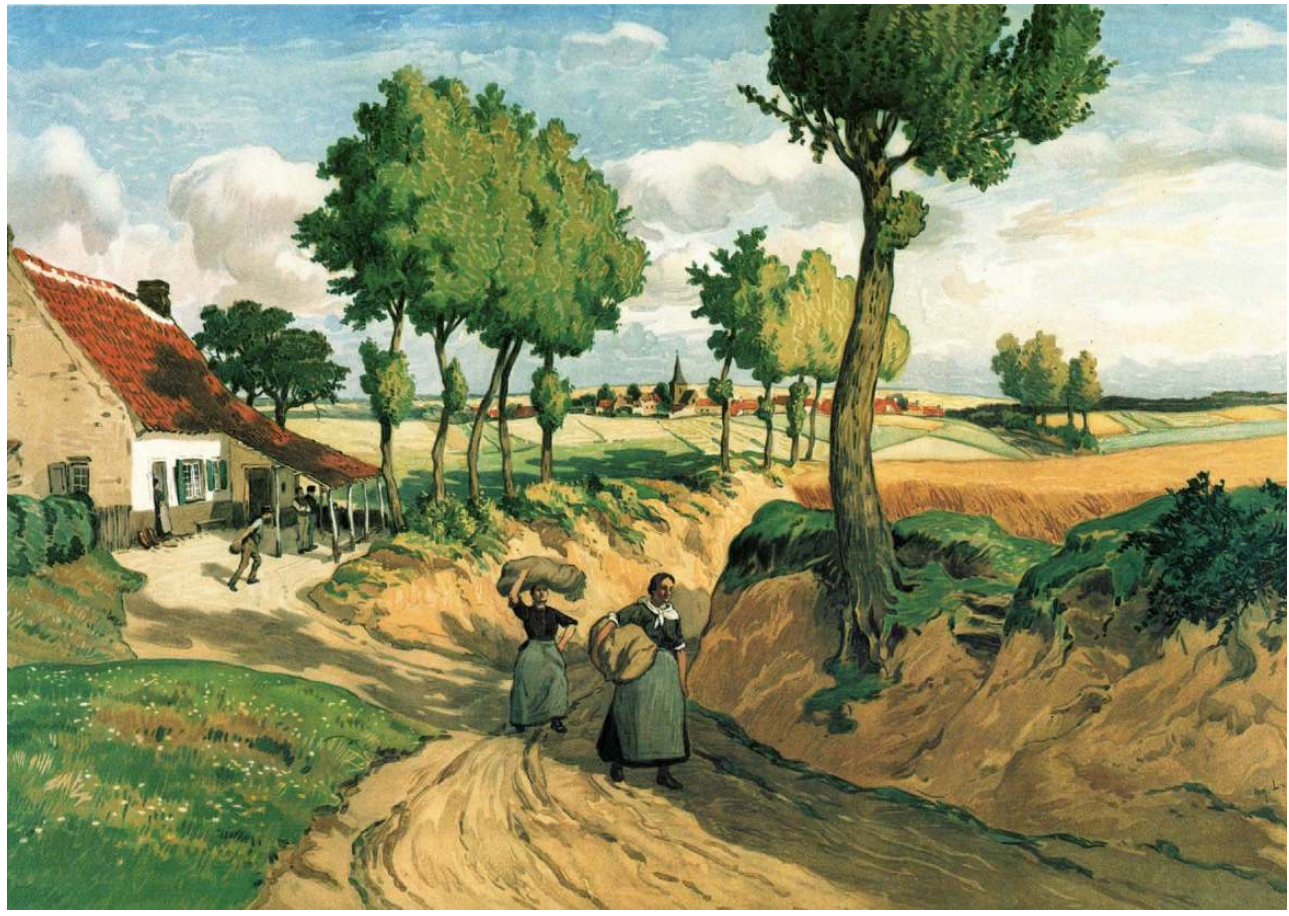




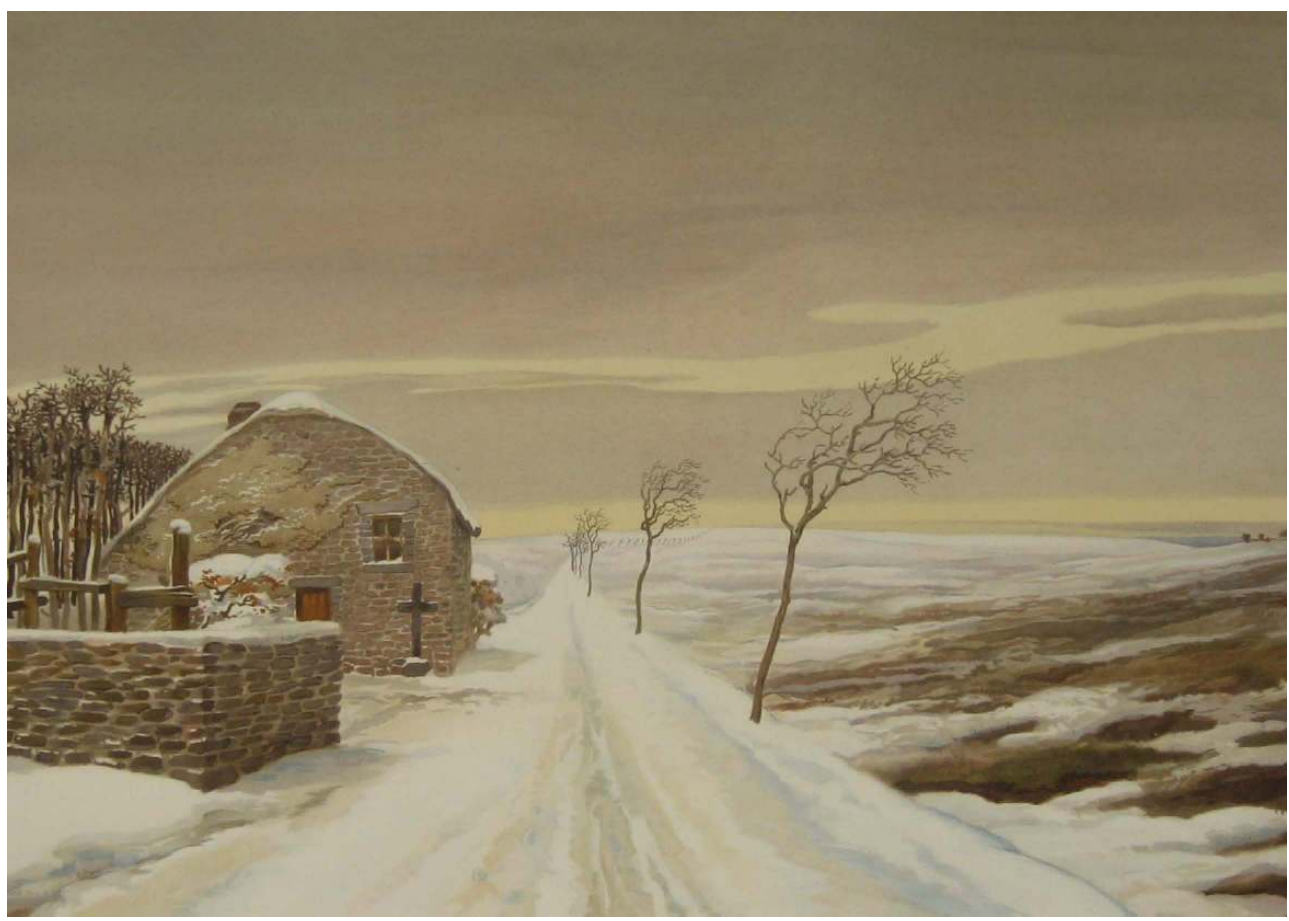

Figure 20. Fernand TOUSSAINT. La Vallée de la Meuse.

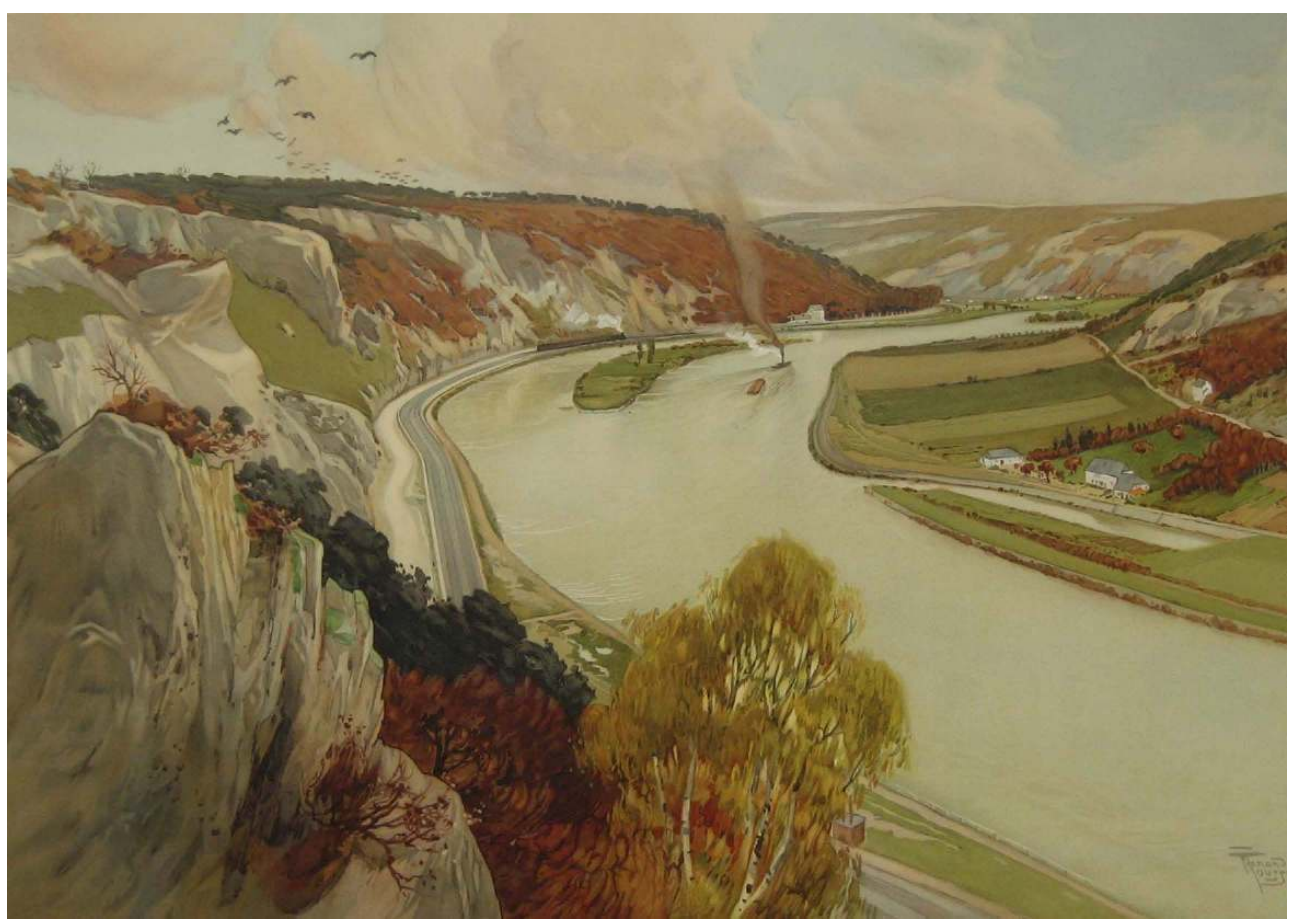


Figure 21. Florimond VAN ACKER. L'Ardenne. Site dans la vallée de l'Ourthe.

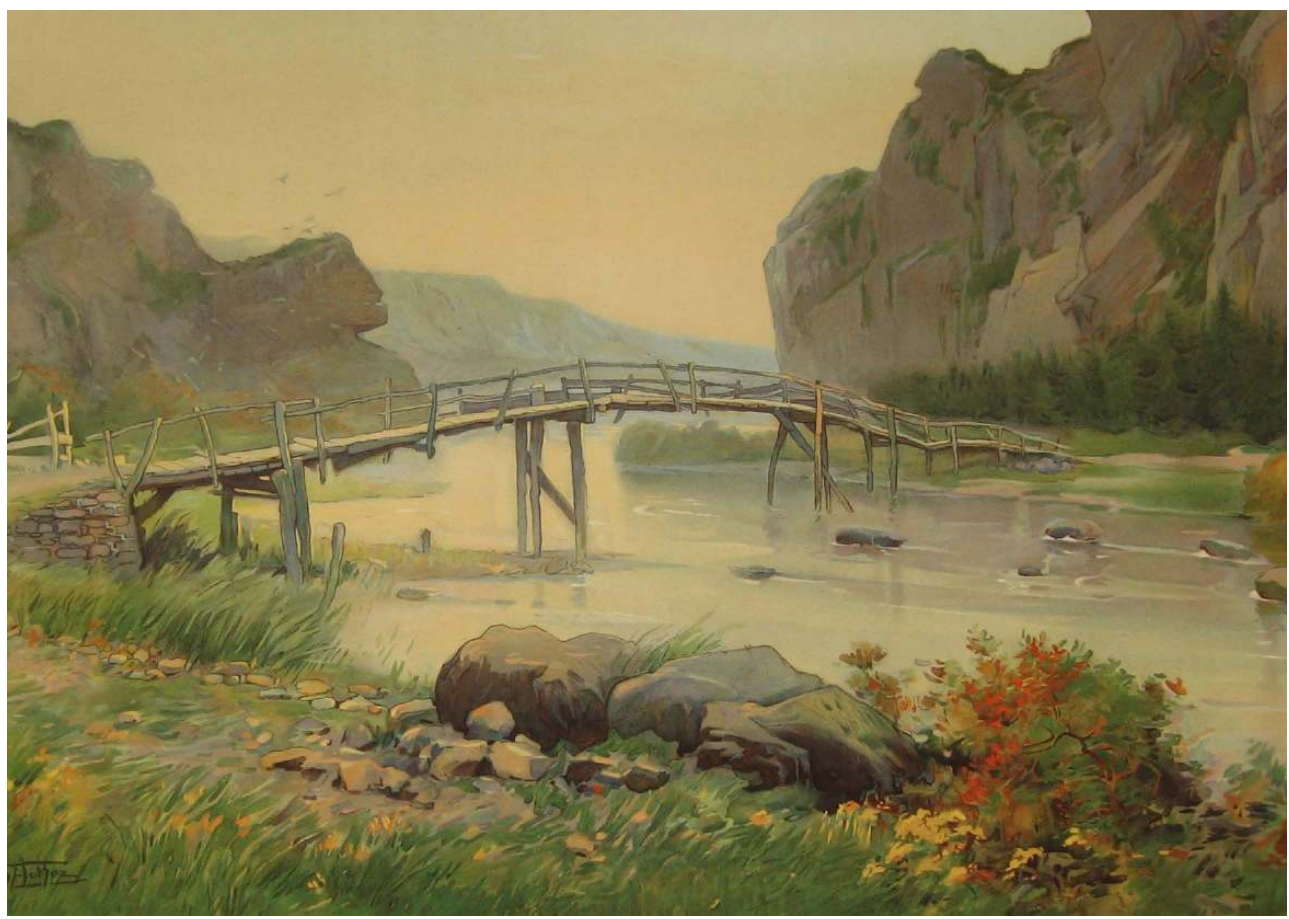

Figure 22. Henri CASSIERS. Le village ardennais.

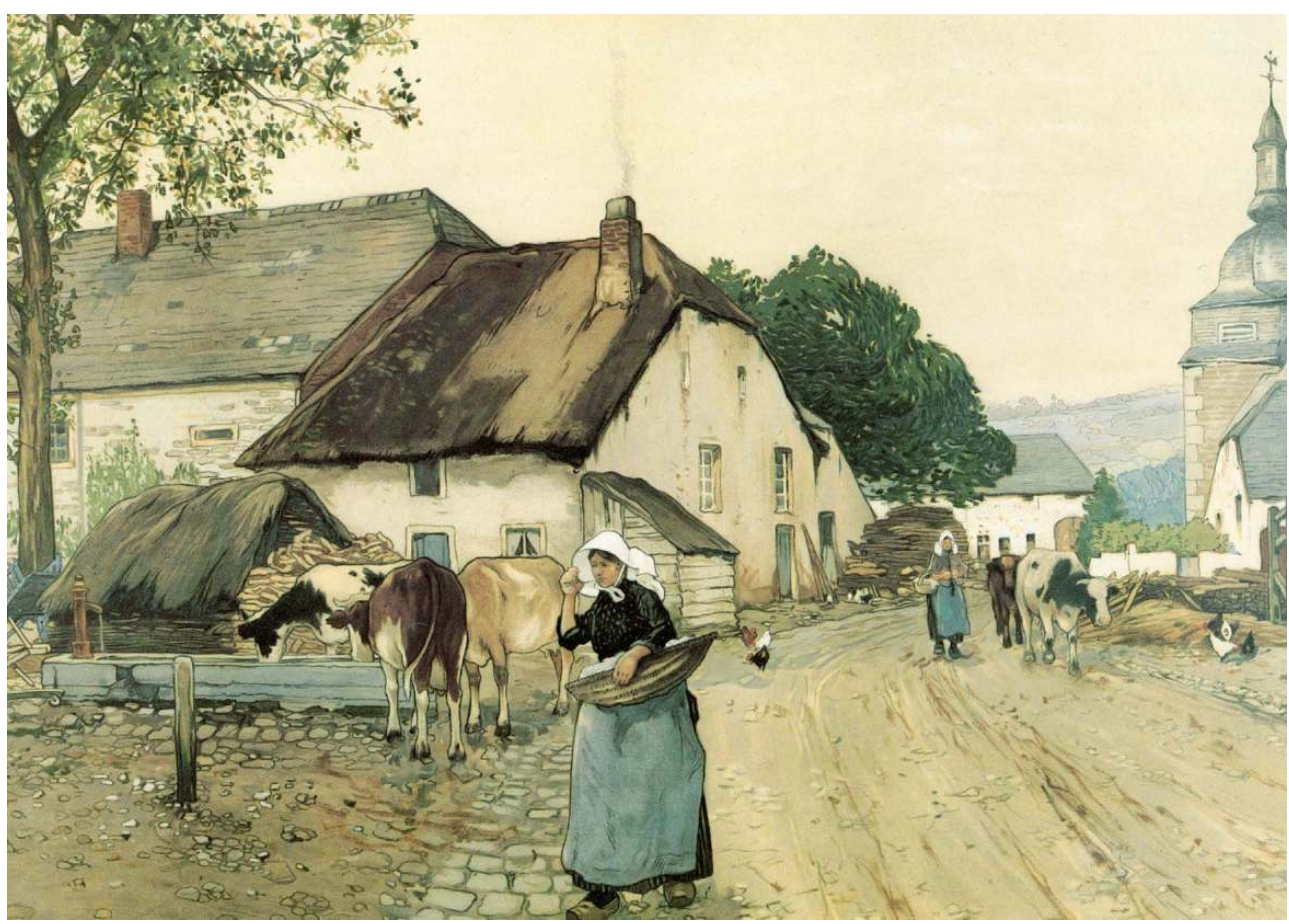

Il est à noter que tous ces artistes, sauf Pierre Paulus, sont d'origine flamande ou bruxelloise, ce que ne manqueront pas de faire remarquer des souscripteurs potentiels de Wallonie (voir plus loin).

La commission semble avoir travaillé très sérieusement pour définir les sites à représenter. C'est bien la connaissance géographique du pays et le souci d'un 
échantillonnage de ses régions qui a guidé la recherche des sujets et les instructions données aux artistes. Si la notion de "vue pittoresque" n'est pas totalement exclue (elle s'exprime certainement dans l'estampe consacrée à Huy), le souci de caractériser chaque paysage est présent dans l'énumération des objets qui doivent figurer dans chaque tableau. Dans le dossier conservé aux archives communales, une enveloppe de fiches manuscrites et plusieurs grandes feuilles récapitulatives insistent sur le sujet à représenter et sur le but à poursuivre, c'est-à-dire sur ce qu'il faut montrer. Il est fait parfois allusion à des photos qui pourraient guider le travail de l'artiste.

Voici quelques exemples de ces instructions, d'après notamment le projet détaillé figurant en annexe de la lettre de l'échevin Léon Lepage du 19 octobre 1906 (AB, IP 2500, dossier 5) . Pour l'estampe "Le Littoral et la Dunes", un bateau échoué doit faire comprendre le mécanisme des marées. Pour l'estampe "Paysage brabançon", il est nécessaire de faire figurer un chemin creux où "se montre encore la flore sauvage, chassée partout par la culture". Pour l'estampe "La Vallée de la Meuse", entre Dinant et Namur, on insistera sur la notion de plateau. On montrera "que la vallée, même large, n'est qu'un accident dans le plateau ardennais" (il s'agit en fait du plateau condruzien); on montrera aussi la différence entre la rive concave rongée par le fleuve et la rive convexe en pente douce. L'estampe "Le Pays industriel" doit comporter un terril, les flammes d'un haut-fourneau, des laminoirs, des ouvriers. On donne en modèle un tableau de J.P. Laurens figurant dans la revue Art et Décoration de 1906. Sans doute à cette époque, Pierre Paulus n'était-il pas encore une référence. Pour l'estampe "La Fagne" (il s'agit des Hautes Fagnes, aux environs de Hockai), il faut que l'immensité de la surface du plateau soit rendue de même que la permanence du vent (on représentera une grande haie de hêtre du côté ouest ou sud-ouest du bâtiment); la rigueur du climat sera exprimée par la couverture neigeuse ; un tas de tourbe mise à sécher fera "comprendre la nature du sol et sa grande humidité" (ce point ne sera pas représenté). La conquête de la fagne par l'agriculture moderne grâce aux engrais devrait être évoquée par la présence d'une ferme. Rien par contre sur les boisements en épicéas qui ont pourtant envahi déjà une bonne partie du plateau.

31 La Commission s'est demandé si certains sujets n'étaient pas répétés, s'il fallait vraiment représenter plusieurs vallées encaissées différentes de Haute-Belgique. Peut-être est-ce pour ce motif qu'a été abandonnée la vue du barrage et du lac de la Gileppe qui a été remplacée par une vue de Huy. On a renoncé aussi, en cours de route, à une estampe qui devait montrer les polders du bas Escaut, en aval d'Anvers.

Certaines critiques peuvent évidemment être portées sur les choix qui ont été faits et parfois aussi sur la pertinence des instructions aux artistes. Le paysage brabançon, qui est demandé, ne correspond pas au Brabant qui s'étend au sud-est de Bruxelles où des vallées bien marquées aux versant boisés entaillent le plateau sableux. La présence d'une fermette de type flamand ferait plutôt penser à un paysage entre Bruxelles et Louvain, donc dans la partie la plus septentrionale du plateau. L'absence de tout paysage condruzien avec son assemblage de prés, de champs et de bois et ses villages de pierre peut étonner. Si la ferme poldérienne est représentée par la grande grange qui figure dans le paysage des environs de Furnes, on n'a demandé aucune grande ferme wallonne à cour carrée ni aucune ferme-château. Le seul exemple de village wallon est le village ardennais. Il est vrai qu'il n'y a pratiquement qu'un seul exemple de village flamand, celui du nord de la West-Flandre. 

de l'époque Les vues de villes flamandes s'inscrivent sous le thème général du déclin et traduisent même une conception anti-moderniste puisqu'elles doivent être présentées comme des exemples de "villes mortes". Ainsi pour la Grand-Place de Furnes, il est demandé à l'artiste d'insister sur l'opulence de son décor qui contraste avec la décadence actuelle. Le même objectif doit être atteint avec la vue de Bruges. L'idée à représenter est à la limite parfois du préjugé. L'estampe d'un village flamand doit montrer les matériaux de construction (briques et tuiles) mais doit aussi insister sur une impression de calme et de propreté. Pour le village ardennais, on parlera aussi des matériaux de construction (pierres et ardoises) mais on n'oubliera pas de montrer les tas de fumier qui donnent une sensation de malpropreté. Le cliché de la propreté du Nord opposée à la malpropreté et au laisser-aller du Sud est bien présent. Que le lecteur se rassure! Les artistes n'ont pas vraiment exprimé cette sensation. rivière (la Sambre), la couleur noire prédominante évoquant le charbon et le fer. Dans l'estampe consacrée à la campagne près de Furnes, tous les éléments demandés sont présents y compris, dans le lointain, la ligne jaune des dunes. Mais les artistes ont parfois pris quelques libertés. On sent ainsi que Henri Cassiers, auteur des grandes affiches de la Red Star Line et d'autres compagnies maritimes, n'a pas résisté au plaisir de dessiner, dans la rade d'Anvers, les proues majestueuses d'un transatlantique et d'un grand voilier sans qu'apparaisse un seul élément de l'équipement portuaire. Seule la tour de la cathédrale dans le lointain identifie le lieu. Mais l'impression de la puissance du port est très forte. L'estampe "Le Littoral et la Dune" a été pour lui l'occasion de peindre une marine. Lynen n'a pas résisté à la tentation de l'anecdote en plaçant un groupe dans son tableau brabançon, des joueurs de quilles devant un estaminet, malgré la réticence des membres de la commission devant tout effet de ce type.

La vallée de la Meuse, entre Dinant et Anhée dans l'estampe de Fernand Toussaint, est bien représentée, comme convenu, encaissée dans le plateau. La vue est prise de la rive gauche, aux environs des ruines du château de Crèvecoeur, à Bouvignes. L'auteur n'a pas manqué d'ajouter, au pied de la rive concave, le détail un peu naïf du train crachant sa fumée et, sur le fleuve, un petit bateau à vapeur remorquant une barge Les rochers par contre sont dessinés d'une façon schématique qui ne rend que très imparfaitement la structure géologique. La vue de Liège, du même artiste, montre sous les pieds de l'observateur les nombreux trains de la gare des Guillemins.

Certains des sites représentés sont certainement inspirés de photographies. C'est le cas pour Huy qui relève de la catégorie des vues pittoresques. La Grand-Place de Furnes, le 
quai de Bruges, la Bourse de Bruxelles sont des reproductions fidèles de sites existants. C'est moins sûr pour le village ardennais et même pour le village de West-Flandre. La campagne de la région de Furnes donne l'impression d'un paysage composé pour rassembler les éléments demandés à l'auteur: la grande grange des polders, la ligne de dunes à l'horizon. C'est l'impression aussi que donnent le paysage industriel de Paulus, la campagne brabançonne de Lynen, la Fagne et même la Campine. Ce sont presque des modélisations. Celles-ci traduisent en tout cas une perception assez sensible des paysages.

Tout compte fait, cette série de 21 estampes exprime fidèlement la conception que l'on avait des paysages de la Belgique du début du $\mathrm{XX}^{\mathrm{e}}$ siècle. Ces paysages sont contemporains de ceux que Paul Vidal de la Blache a décrits en 1908 dans son tableau géographique de la France, c'est-à-dire des "paysages traditionnels", produits finis d'une évolution séculaire (depuis le moyen âge au moins) avec les retouches apportées par la révolution industrielle. Ces aboutissements d'un temps long qui respectaient une typologie régionale très marquée pouvaient apparaître alors comme des monuments immuables.

Ces estampes expriment aussi la vision que la bourgeoisie belge du début du $\mathrm{XX}^{\mathrm{e}}$ siècle avait de son pays. Les artistes sont d'ailleurs des membres de cette petite bourgeoisie. Dans les paysages urbains, les scènes de marché, avec leur clientèle bourgeoise, sont privilégiées. La vue des boulevards centraux à hauteur de la Bourse est une belle mise en scène de la bourgeoisie bruxelloise. Dans les vues de villages, ce sont souvent des paysannes et des paysans idéalisés avec leurs costumes et leurs coiffes. La dureté des travaux campagnards n'est pas exprimée. A ce sujet, des remarques ont été faites sur les paysans et paysannes de Henri Cassiers qui sont souvent très nombreux dans ses chromolithographies de villages flamands ou hollandais ou dans ses affiches publicitaires. Ils semblent mener une vie de loisirs et de non-activité et représentent une population toujours saine et joyeuse (A. Reyniers, 1994. p. 101) répondant plutôt aux canons d'une population nantie. Quant aux dures conditions de vie des ouvriers industriels, elles n'apparaissent pas, contrairement à ce qu'on aurait pu attendre de cet artiste, dans le tableau de Pierre Paulus puisqu'il nous donne une vue à vol d'oiseau, purement "minérale" d'un complexe sidérurgique où les hommes ne sont pas visibles.

41 On remarquera enfin que tous les paysages représentés sont bien des paysages "géographiques", c'est-à-dire des paysages aménagés par l'homme. Contrairement à d'autres collections de paysages de l'époque, comme les vues photographiques de Jean Massart en géographie botanique, on n'a pas voulu retenir les paysages "naturels". Seule la vallée de l'Ourthe, sans doute près du Hérou, pourrait être considérée comme telle, avec comme unique marque humaine, la passerelle enjambant la rivière. Lorsqu'on envisagea un moment de représenter le lac de la Gileppe, il est évident que c'est à un exemple de paysage fabriqué par l'homme que l'on songeait.

La réalisation de la série des 21 estampes exigeait la vente d'un millier de séries afin que le prix fût suffisamment bas pour des établissements scolaires ou du moins pour les autorités dont ils dépendaient. Des dizaines, sinon des centaines, de lettres souvent personnalisées furent envoyées par le Collège de la Ville de Bruxelles, généralement par l'entremise de l'Echevin de l'Instruction publique, à des souscripteurs potentiels, par exemple aux Collèges échevinaux, aux pouvoirs organisateurs des différents réseaux d'enseignement, à différents ministres (voir $A B$, dossier IP II 2306, c et d). L'argumentation variait selon le destinataire. Pour les différents réseaux d'enseignement, la valeur didactique était mise en avant tout autant que la qualité artistique. Si le but 
principal évoqué était la décoration des locaux scolaires et l'éducation esthétique, particulièrement dans les localités où il n'y avait pas de musées, les estampes étaient présentées aussi comme d'excellents documents pour le cours de géographie et comme matière à de nombreuses leçons de langue par la méthode directe. L'utilité de tels documents était particulièrement soulignée pour les écoles normales où l'enseignement de la géographie de la Belgique tenait une part importante. Au Ministre des Chemins de fer, Poste et Télégraphe, les estampes étaient présentées comme des éléments qui pourraient orner les gares et qui en même temps attireraient l'attention des touristes sur la diversité et la beauté des régions du pays. Au Ministre de l'Industrie et des Travaux, cette collection aurait dû permettre de montrer que l'industrie belge était capable de rivaliser, sinon de surpasser, dans ce domaine, les réalisations des autres pays. On ira même jusqu'à écrire que ces estampes formeraient non seulement des hommes meilleurs mais en outre "des ouvriers mieux doués pour lutter, dans l'industrie, contre la concurrence étrangère"! La firme éditrice a elle-même lancé une brochure publicitaire à destination du grand public dans laquelle elle affirme que, dans les habitations, ces estampes embelliront le home et le rendront plus familier. Les estampes pourront d'ailleurs être vendues à la pièce, au prix de 10 francs.

Nous ne savons pas quelle a été la réussite de la souscription mais elle semble avoir été suffisante pour que la série fût réalisée. Certaines administrations communales de l'agglomération de Bruxelles -Schaerbeek et Saint-Gilles notamment- ont souscrit généreusement. La ville d'Anvers a commandé de nombreuses séries (50 peut-être $60 ; A B$, dossier IP II 2306, c). Gand et Mons semblent avoir été aussi de bons clients.

Mais il y eut de fortes réticences dans la partie wallonne du pays. Une longue correspondance a été échangée ainsi avec le Collège des Bourgmestre et Echevins de Liège. Ce dernier a constaté en effet, dans une lettre du 20 octobre 1906 (AB, dossier IPII 2306, c) que "les artistes recommandés pour la confection des modèles, sont exclusivement de Bruxelles, les artistes wallons sont ainsi sacrifiés au profit des peintres de la région flamande du pays" Il ajoute : "Nous ne comprendrions pas, par exemple, que celui qui n'a jamais oeuvré qu'en Flandre, fût chargé de nous rendre l'Ardenne, la vallée de la Meuse ou celle de l'Ourthe ! Pour rendre avec sincérité le caractère d'une contrée, il faut y avoir vécu".

La ville de Mons estime que la province du Hainaut n'est pas suffisamment représentée. On n'y trouve que Thuin mais pas Tournai ni Mons. Avant de souscrire, il faudrait que cette lacune fût comblée.

Un incident se produisit avec la Ville d'Anvers lorsque le peintre qu'elle avait proposé pour une vue depuis la rive gauche, Van Neste, ne fut pas retenu par la commission. Anvers se montra d'autant plus amère qu'elle avait été un gros souscripteur.

Comparées aux illustrations des manuels de l'époque, les estampes ont assurément une valeur didactique et esthétique bien supérieure. Dans l'atlas d'illustrations (Le Tour $d u$ Monde en Images), publié par Alexis M.G.(1909), le grand auteur de manuels scolaires, complétant son atlas scolaire proprement dit, le Tour de Belgique fait 16 pages de 4 à 6 illustrations chacune mais comporte surtout des gravures faites à partir de vues photographiques de sites pittoresques et de monuments de tous types, de statues de personnages célèbres, avec parfois des dessins (dunes, houblonnières, tabac, haut fourneau, coupe d'un charbonnage, etc.) mais fort peu de paysages géographiques proprement dits, les paysages ruraux étant curieusement quasi absents (figure 23). Les vues sont souvent de trop petites dimensions pour que les élèves puissent en faire une 
analyse détaillée. Ce qui n'est pas le cas des estampes qui ont été conçues pour être accrochées au mur et non pour être reproduites dans un livre scolaire.

Figure 23. Une des pages consacrées à la province d'Anvers dans “Le Tour du Monde en Images. Album géographique" d'Alexis-M.G., édition 1909. Comparer la vue du port d'Anvers avec celle de H. Cassiers.

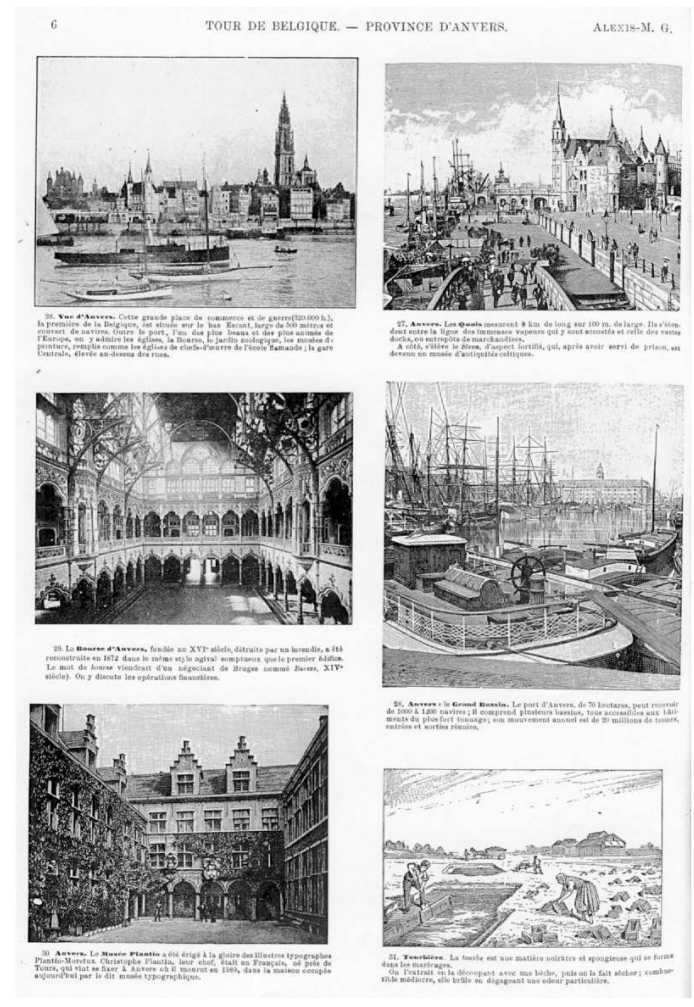

Il est indéniable que ces estampes ont du charme. Traitées dans des couleurs chaudes, elles ont la clarté de cartes postales illustrées. Elles évoquent parfois les images en couleurs données en prime dans les emballages de chocolats ou qui décorent les boîtes de biscuits. Un paysage mosan de Florimond Van Acker, qui ne figure pas dans la série, mais qui est d'une facture très semblable, a été utilisé ainsi par la firme De Beuckelaer, d'Anvers (Cabinet des Estampes, SI 4665).

En 1921, Marius Renard, directeur de l'Institut des Arts et Métiers de Saint-Ghislain et homme politique socialiste bruxellois, a consacré à la série - celle-ci était alors encore en vente - un article particulièrement élogieux dans un des premiers numéros de Savoir et Beauté, une sympathique revue mensuelle qu'il dirigeait avec Paul Pastur et dont un des objectifs était de diffuser l'art dans les écoles. Pour lui, cette série d'estampes était unique au monde et répondait parfaitement aux idées exprimées par différents pédagogues, dont des auteurs anglais d'estampes du même type, pour lesquels les images devaient être simples, rechercher la vitalité plutôt que le réalisme et mettre l'effet dans l'emploi de tons plats et de contours accusés (M.Renard, 1921, p. 150). L'estampe de Cassiers sur le village du nord de la West-Flandre, que Marius Renard reproduit en couleurs dans son article, lui apparaît comme le modèle même de l'image scolaire murale. "Tout, dans cette oeuvre remarquable, écrit-il, peut concourir à l'enseignement en plus de la beauté : le sujet, les personnages, les maisons, les arbres, etc. Il y a là nombre de sujets de questions..." (M.Renard, 1921, pp. 151-153). 
Que sont devenues ces estampes? Il parait très difficile aujourd'hui d'en trouver une série complète, à l'exception de celle qui se trouve au Cabinet des Estampes de la Bibliothèque Royale et vraisemblablement dans des collections privées. On n'en trouve pas trace - ou en tout cas les autorités actuelles n'en ont conservé aucun souvenir - dans les institutions publiques. Beaucoup ont disparu, semble-t-il, lors de la destruction, de la rénovation ou du déménagement de l'école dans laquelle elles se trouvaient. Quelques-unes ont été préservées parce qu'elles ornaient un bureau de direction ou un local de réception. En 1984, une firme pétrolière en a reproduit une dizaine dans le calendrier offert à ses clients. Les chromolithographies d'Henri Cassiers ont figuré dans l'exposition organisée à l'occasion du cinquantenaire de sa mort, à Anvers et à Katwijk (Pays-Bas).

La série mériterait certainement d'être rassemblée dans une exposition qui montrerait au public d'aujourd'hui l'image que les Belges avaient de leur pays au début du XXe siècle. Elle rappellerait aussi un exemple intéressant de l'utilisation de la géographie dans un souci d'éducation esthétique, en partant des paysages modelés par l'homme, ce qui est rafraîchissant à une époque où des géographes disent trop souvent que "la géographie, ça sert d'abord à faire la guerre".

\section{BIBLIOGRAPHIE}

Archives de la Ville de Bruxelles, boîtes IP II 2300 et 2306.

ALEXIS M.G. (1909), Le Tour du Monde en Images. Album géographique, Liége, H. Dessain, Paris, Procure générale, Alost, Procure des Frères, $9^{e}$ édition (la première édition est de 1899).

Dictionnaire biographique illustré des Artistes de Belgique depuis 1830 (1995), Bruxelles, Arto.

Henri Cassiers 1858-1944 (1994), Antwerpen, Museum Vleeshuis (1 avril-12 juin 1994), Katwijk, Katwijks Museum, 25 juin-24 septembre 1944, Antwerpen, Pandora, 166 p.

L'éducation esthétique à l'école primaire (1903), Ville de Bruxelles, 30 p. (Archives de la Ville de Bruxelles, dossier IP II 2300, 1).

KOECKELBERGH I. et POTTIER W. (1994), “Henri Cassiers et la Belgique”, dans Henri Cassiers, Antwerpen, Pandora, pp. 9-13.

POTTIER W. (1994), “Les planches didactiques d'Henri Cassiers”, dans Henri Cassiers, Antwerpen, Pandora, pp. 124-129.

RENARD M. (1921), “Les Estampes Décoratives Scolaires”, Savoir et Beauté, 1 ère année, $\mathrm{n}^{\circ} 5$, octobre 1921, Saint-Ghislain, Bruxelles, pp. 147-153 (22 illustrations dont 2 planches en couleurs).

REYNIERS A. (1994), “Les idéologies du sexe et de l'image dans l'univers publicitaire de Henri Cassiers", dans Henri Cassiers, Antwerpen, Pandora, pp. 97-103.

SLUYS A. (1907), “Imagerie scolaire. Suède. Japon. Belgique”, L'Art Public, Revue de l'Institut International de l'Art Public, 1, pp. 56-60.

STENGERS J. et GUBIN E. (2002), Histoire du sentiment national en Belgique des origines à nos jours. Tome 2 : le grand siècle de la nationalité belge, Bruxelles, Racine, $234 \mathrm{p}$. 


\section{AUTEUR}

\section{HENRI NICOLAÏ}

Université Libre de Bruxelles, henri.nicolai@scarlet.be 